\title{
Intranasal Oxytocin and Vasopressin Modulate Divergent Brainwide Functional Substrates
}

\author{
Alberto Galbusera', Alessia De Felice', Stefano Girardi², Giacomo Bassetto², Marta Maschietto², \\ Katsuhiko Nishimori ${ }^{3}$, Bice Chini ${ }^{4,5}$, Francesco Papaleo ${ }^{6}$, Stefano Vassanelli ${ }^{2}$ and Alessandro Gozzi ${ }^{*, 1}$ \\ 'Functional Neuroimaging Laboratory, Center for Neuroscience and Cognitive Systems @ UniTn, Istituto Italiano di Tecnologia, Rovereto (TN), Italy; \\ ${ }^{2}$ Department of Biomedical Sciences, University of Padova, Padova, Italy; ${ }^{3}$ Laboratory of Molecular Biology, Department of Molecular and Cell \\ Biology, Graduate School of Agricultural Science, Tohoku University, Miyagi, Japan; ${ }^{4}$ CNR, Institute of Neuroscience, Milan, Italy; ${ }^{5}$ Department of \\ Biotechnology and Translational Medicine, University of Milan, Milan, Italy; ${ }^{6}$ Department of Neuroscience and Brain Technologies, Istituto Italiano di \\ Tecnologia, Genova, Italy
}

\begin{abstract}
The neuropeptides oxytocin (OXT) and vasopressin (AVP) have been identified as modulators of emotional social behaviors and associated with neuropsychiatric disorders characterized by social dysfunction. Experimental and therapeutic use of OXT and AVP via the intranasal route is the subject of extensive clinical research. However, the large-scale functional substrates directly engaged by these peptides and their functional dynamics remain elusive. By using cerebral blood volume (CBV) weighted $\mathrm{AMRI}$ in the mouse, we show that intranasal administration of OXT rapidly elicits the transient activation of cortical regions and a sustained activation of hippocampal and forebrain areas characterized by high oxytocin receptor density. By contrast, intranasal administration of AVP produced a robust and sustained deactivation in cortico-parietal, thalamic and mesolimbic regions. Importantly, intravenous administration of OXT and AVP did not recapitulate the patterns of modulation produced by intranasal dosing, supporting a central origin of the observed functional changes. In keeping with this notion, hippocampal local field potential recordings revealed multi-band power increases upon intranasal OXT administration. We also show that the selective OXTderivative TGOT reproduced the pattern of activation elicited by OXT and that the deletion of OXT receptors does not affect AVP-mediated deactivation. Collectively, our data document divergent modulation of brainwide neural systems by intranasal administration of OXT and AVP, an effect that involves key substrates of social and emotional behavior. The observed divergence calls for a deeper investigation of the systemslevel mechanisms by which exogenous OXT and AVP modulate brain function and exert their putative therapeutic effects.
\end{abstract}

Neuropsychopharmacology (2017) 42, I420-1434; doi:I0.I038/npp.2016.283; published online I8 January 2017

\section{INTRODUCTION}

Oxytocin (OXT) and arginine-vasopressin (AVP) are two closely related neuropeptides known for their prominent role in mammalian social cognition and behavior (Donaldson and Young, 2008). Animal studies showed that brain synthesis and release of OXT and AVP are driven by positive and negative social stimuli, involving the concerted modulation of multiple brain systems (Huber et al, 2005; Donaldson and Young, 2008; Yoshida et al, 2009; Stoop, 2014). These features, along with accumulating evidence of altered OXT and AVP signaling in mental disorders characterized by social dysfunction (eg, autism, schizophrenia) have prompted clinical research into the use of intranasal OXT or AVP administration as a potential treatment for social impairments associated with these

* Correspondence: Dr A Gozzi, Functional Neuroimaging Laboratory, Center for Neuroscience and Cognitive Systems @UniTn, Istituto Italiano di Tecnologia, Rovereto (TN) 38068, Italy, Tel: +39 0464802870।, E-mail: alessandro.gozzi@iit.it

Received 29 March 2016; revised 25 November 2016; accepted 13 December 2016; accepted article preview online 20 December 2016 conditions (Meyer-Lindenberg et al, 2011; Young and Barrett, 2015; Feifel et al, 2016; Guastella and Hickie, 2016). Because peripheral administered OXT and AVP do not cross the blood-brain barrier, human experimental investigations of these neuropeptides entails their intranasal administration (Born et al, 2002). However, despite accumulating clinical research into the therapeutic potential of exogenous OXT and AVP administration with some initial encouraging results (Bakermans-Kranenburg and van IJzendoorn, 2013; Hofmann et al, 2015), our knowledge of the brainwide substrates engaged by exogenous intranasal administration of these two neuropeptides remains limited (Leng and Ludwig, 2016; Walum et al, 2016).

Functional magnetic resonance imaging (fMRI) studies in humans have examined the indirect modulatory effect of intranasal OXT or AVP on patterns of brain activity elicited by emotionally or socially relevant stimuli engaging specific brain targets (Zink and Meyer-Lindenberg, 2012; Jocham Radua and Shergill, 2015). These task-based fMRI investigations have provided evidence of a modulatory effect of OXT on the activity of the amygdala in response to a variety of stimuli (Kirsch et al, 2005; Meyer-Lindenberg et al, 2011), 
along with the possible modulation of cortico-limbic substrates (Sripada et al, 2013; Riem et al, 2014) and mesolimbic regions of the reward system (Baumgartner et al, 2008). Task-based fMRI investigations of AVP have been much fewer and have so far revealed a possible involvement of cingulate and temporal-parietal regions, along with a modulation of caudate nuclei and the amygdala (Zink and Meyer-Lindenberg, 2012). However, task-based neuroimaging approaches only unmasks modulatory effects uniquely confined to the neural network engaged by the task and that take place on top (or in spite) of the functional response elicited by the stimulus per se. As a result, the macroscale substrates directly engaged by intranasal administration of OXT and AVP may be more widespread and pervasive than what so far described with task-based imaging. This hypothesis is consistent with the results of a recent task-free imaging study in humans, where intranasal OXT produced a previously unreported engagement of subcortical limbic regions (eg, the hippocampus) and basal forebrain nuclei (Paloyelis et al, 2014). Preliminary rodent imaging studies upon intraventricular administration of OXT support the notion that the substrates affected by this neuropeptide may be distributed and involve multiple brain systems (Ferris et al, 2015).

Collectively, these initial imaging studies suggest that intranasal OXT and AVP may exert their action via the modulation of key central effectors of social and emotional behavior. However, the specific substrates engaged by these peptides, as well as their functional dynamics, remain elusive. Moreover, the relative contribution of OXT and AVP receptors in modulating macroscale functional activity and the direction of their modulatory effect remain largely unknown. A disambiguation of these aspects may also help to understand some conflicting reports as to the central effect of exogenously administered OXT and AVP in rodents. Indeed, despite considerable overlap in the pharmacological profile, molecular signaling and electrophysiological responses elicited by these two neuropeptides (Raggenbass, 2001; Stoop, 2014), and the largely prevailing view of a concerted contribution of these two systems to affiliative (Ross and Young, 2009), social recognition (Insel, 2010; Lukas and Neumann, 2013) and maternal behavior (Bosch et al, 2010), pharmacological manipulations in rodents have highlighted a differential modulatory effect of OXT and AVP on specific behavioral domains (Neumann and Landgraf, 2012; Stoop, 2012). For example, exogenous OXT administration produces anxiolytic and stress-protective effects (McCarthy et al, 1996; Windle et al, 1997; Windle et al, 2004), while loss-of-function studies of AVP empirically support an anxiogenic role of this peptide system (Aguilera et al, 1994; Ryckmans, 2010). Similarly, brain AVP in rodents appears to modulate depression-like behavior in an opposite manner to OXT, resulting in a shift toward passive stresscoping (Landgraf et al, 2007; Neumann and Landgraf, 2012; Neumann and Slattery, 2016). Moreover, acute intranasal OXT exhibits prosocial effects in humans and rodents (Lukas et al, 2011; Meyer-Lindenberg et al, 2011; Huang et al, 2014) but synthetic intranasal AVP in humans produces increased autonomic and behavioral responses to threatening stimuli (Ebstein et al, 2009; Shalev et al, 2011), and selective stimulation of $\mathrm{V} 1 \mathrm{~b}$ receptor in rodents produces antisocial effects (Litvin et al, 2011). Divergent microcircuital effectors have been identified for AVP and OXT in specific brain regions (ie, the amygdala, Huber et al, 2005); however, the broader and brainwide mechanisms by which these two systems can produce convergent or competing behavioral outcomes remain undetermined. A deeper investigation of these aspects is critically required to better assess the mechanism of action and putative therapeutic scope of OXT and AVP in human psychiatric disorders.

To begin to investigate these issues, we used high-resolution pharmacological fMRI (phMRI; Ferrari et al, 2012) to map the functional dynamics and brainwide functional substrates engaged by intranasal OXT and AVP in the mouse brain. Our measurements rely on the use of intravascular iron oxide nanoparticles to sensitize images to relative cerebral blood volume (rCBV), an approach that results in increased functional contrast to noise (Mandeville et al, 1998), reduced susceptibility artefacts (Sforazzini et al, 2014), and that is characterized by a more direct relationship with underlying neuronal activity with respect to traditional BOLD fMRI (Schridde et al, 2008). Our main finding is that, despite their evolutionary and neurobiological similarity, OXT and AVP exert opposing brainwide modulatory effects involving, respectively, the activation and deactivation of large functional networks, including key rodent substrates of social and affective behavior. Importantly, intravenous administration of OXT and AVP did not recapitulate the patterns of modulation produced by intranasal dosing, supporting a central origin of the observed functional response. The observation of a functional divergence, rather than a concordant modulatory action, calls for a deeper investigation of the systems-level mechanisms by which exogenous OXT and AVP modulate brain function and exert their putative therapeutic effects.

\section{MATERIALS AND METHODS}

A more detailed description of the methods employed can be found in the Supplementary Method section. All research involving animals were carried out in accordance with the Italian Legislative Decree no. 116, 27 January 1992.

\section{Experimental Subjects}

Adult male C57BL/6J mice (13-24 weeks) were used throughout the study unless otherwise specified. OXT- $\mathrm{R}^{-1-}$ and littermate control wild-type OXT- $\mathrm{R}^{++}$mice (Takayanagi et al, 2005) rederived on a C57BL/6J genetic background (Charles Rivers, Calco, Italy), were maintained by heterozygous mating.

\section{Drug Formulation}

Oxytocin, [Thr4,Gly7]-Oxytocin (TGOT) and [Arg8]-Vasopressin (Sigma Aldrich, Milan), were dissolved in distilled water at a concentration of $333.4 \mu \mathrm{g} / \mathrm{ml}$. Distilled water was used as vehicle in all the intranasal control groups. For intravenous fMRI studies, peptides were dissolved in physiological saline solution.

\section{Functional Magnetic Resonance Imaging}

Animal preparation for fMRI has been previously described in great detail (Sforazzini et al, 2014). Mice were imaged under halothane sedation $(0.8 \%)$. Ventilation parameters 
were adjusted to maintain arterial $\mathrm{p}_{\mathrm{a}} \mathrm{CO}_{2}$ levels $<40 \mathrm{~mm} \mathrm{Hg}$ and $\mathrm{paO}_{2}$ levels $>90 \mathrm{~mm} \mathrm{Hg}$ corresponding to $98 \%$ hemoglobin saturation (Supplementary Table S1). fMRI data were acquired using a $7 \mathrm{~T}$ Pharmascan (Bruker, Ettlingen, Germany). Images were sensitized to reflect alterations in rCBV using $5 \mu \mathrm{l} / \mathrm{g}$ of blood-pool contrast agent (Errico et al, 2015).

\section{Intranasal Administration}

To allow for the intranasal administration of drugs inside the scanner, an intranasal catheter was inserted into each nostril as previously described (Donnelley et al, 2009). An Hamilton Syringe $710 \mathrm{~N} 100 \mu \mathrm{l}$ was coupled with two PTFE cannulas 1 Fr (one for each nostril) using a $60 \mathrm{~cm}$-long PE10 catheter connected to a sterile Y connector $(22 \mathrm{G})$. Standard silicon laboratory tubing was used for all coupling connections. The syringe was then filled with distilled water. Four microliters of air $(2 \mu \mathrm{l}$ per each end of the intranasal cannula) were sucked into the cannulas to avoid mixing with the peptide solution to be administered. To avoid incidental preadministration solution leakage, two additional microliters of air were also sucked in the terminal end of the cannula after compound loading. The two prefilled cannulas were then inserted $9 \mathrm{~mm}$ into the nostrils. Fifteen minutes after contrast agent administration, each subject received an intranasal administration of vehicle or drug for a total volume of $4 \mu \mathrm{l}$ (ie, $2 \mu \mathrm{l}$ of a solution containing $333.4 \mu \mathrm{g} / \mathrm{ml}-$ high dose-or $83.35 \mu \mathrm{g} / \mathrm{ml}$-low dose-of peptide for each nostril). The choice of doses is discussed below. The fMRI response was mapped and quantified as previously described (Squillace et al, 2014).

The two intranasal OXT doses tested $(0.33 \mu \mathrm{g}$ and $1.33 \mu \mathrm{g} /$ mouse) were selected based on previous behavioral studies in the mouse (Huang et al, 2014) and on pilot fMRI tests performed in the laboratory. One international unit (IU) of OXT solution corresponds to $1.667 \mu \mathrm{g}$, so the doses tested corresponded to 0.8 and 0.2 IU per mouse, respectively. Assuming an average weight of ca. $30 \mathrm{~g}$ for an adult male mouse, the low and high OXT dose administered correspond to 6.7 and $26.7 \mathrm{IU} / \mathrm{kg}$, respectively. This dosing is in line with that employed in recent behavioral tests employing acute intranasal OXT administration (eg, 3 and $10 \mathrm{IU} / \mathrm{kg}$, Huang et al, 2014) and significantly lower than the dose used by other rodent investigators in mice, eg, $120 \mathrm{IU} /$ $\mathrm{kg}$ in Penagarikano et al (2015) or $240 \mathrm{IU} / \mathrm{kg}$ in Neumann et al (2013) and Kosaki and Watanabe (2016).

TGOT and AVP are nonapeptides structurally very similar to OXT, with respect to which they differ for two aminoacidic substitutions (Supplementary Figure S1). The intranasal dose of TGOT and AVP tested ( $1.33 \mu \mathrm{g}$ per mouse) was selected out of pilot fMRI studies and replicates the highest amount of OXT examined, which was chosen as a qualitative term of comparison. fMRI mapping of lower TGOT or AVP doses $(0.3 \mu \mathrm{g}$ per mouse) resulted in a more variable functional effects, while at a dose of $0.1 \mu \mathrm{g}$ per mouse both drugs produced subthreshold functional modulation (data not shown). A direct qualitative comparison of the effect of these nonapeptides can be carried out under the assumption of a similar steric hindrance and analogous passive distribution of these peptides into the central nervous system (CNS).

\section{Intravenous fMRI Studies}

To rule out a peripheral origin of the intranasal fMRI changes mapped, we also performed fMRI studies employing peripheral peptide administration. In these experiment, 30 min after contrast agent administration each subject received an intravenous injection of vehicle (saline, which served as reference rCBV baseline for the effects of each neuropeptide) or peptide (OXT TGOT and AVP, doses discussed below), with a total injection volume of $100 \mu$ l. The intravenous fMRI response was quantified in regions of interest as previously described (Squillace et al, 2014).

fMRI studies employing peripheral peptide administration were carried out using the following peptides doses: OXT, $0.03 \mu \mathrm{g} /$ mouse $(n=6) ; \mathrm{TGOT}, 0.03 \mu \mathrm{g} /$ mouse, $(n=6) ; \mathrm{AVP}$, $0.003 \mu \mathrm{g} / \mathrm{mouse},(n=6)$; and four subjects challenged with saline were used as reference controls. The tested doses largely exceed the amounts of peripherally circulating peptides measured upon intranasal administration of high OXT or AVP (described in Results section). Specifically, the OXT dose is $\sim 750$-fold higher the mean circulating OXT level measured in intranasally treated animals, while the AVP dose tested corresponds to $\sim 30$-fold this amount. We tested a lower AVP dose, because in pilot studies, $0.03 \mu \mathrm{g} / \mathrm{mouse}$ of AVP produced in individual animals blood pressure responses exceeding autoregulation range (eg, $130 \mathrm{~mm} \mathrm{Hg}$, Gozzi et al, 2007), hence possibly contaminating central fMRI signals. TGOT was tested at the same dose of OXT, assuming that the two structurally related peptides could result in similar nose-to-vascular translocation.

\section{Experimental Design and Pharmacological Treatments}

fMRI studies were planned based on estimates of intersubject variability and effect size obtained in similar mouse fMRI experiments performed in our laboratory in mice where vehicle and pharmacologically treated groups were employed (Ferrari et al, 2012; Squillace et al, 2014; Errico et al, 2015). Two separate intranasal studies were carried out. Study 1: C57B16/J mice were randomly assigned to the following treatment groups. (a) Intranasal treatment with OXT high dose $(1.33 \mu \mathrm{g} /$ mouse, $n=8)$ (b) intranasal administration of OXT low dose $(0.33 \mu \mathrm{g} / \mathrm{mouse}, n=9)$, (c) intranasal administration of TGOT $(1.33 \mu \mathrm{g} /$ mouse, $n=13)$, and (d) intranasal administration of vehicle (water, $n=7$ ). Study 2: intranasal AVP $(1.33 \mu \mathrm{g} /$ mouse $)$ was tested in a separate experiment in which control $\left(\mathrm{Oxtr}^{+/+}, n=6\right)$ and oxytocin receptor null mice $\left(\mathrm{Oxtr}^{-/-}, n=5\right)$ were used. The effect of AVP was mapped with respect to vehicle baseline in control mice (water, $n=7$ ) under the assumption that intranasal water administration does not significantly alter rCBV baseline (Squillace et al, 2014; Errico et al, 2015). We defined intravenous administration fMRI studies as Study 3 (Supplementary Table S1).

\section{Hippocampal Local Field Potential (LFP) Measurements}

To corroborate a neural origin of the parenchymal signal changes mapped in OXT-treated mice, acute LFP measurements were performed on a separate cohort of anesthetized control mice $(\mathrm{C} 57 \mathrm{~B} 16 / \mathrm{J})$ using the same experimental conditions and anesthetic regimen employed for fMRI. 
Surgery and recordings were performed on a standard stereotaxic apparatus as previously described (Mahmud et al, 2011). OXT $(1.33 \mu \mathrm{g} / \mathrm{mouse}, N=3)$ or vehicle (water, $N=5$ ) were administered after $15 \mathrm{~min}$ of baseline acquisition, and hippocampal activity was recorded for $25 \mathrm{~min}$. Homemade MATLAB (The MathWorks) scripts were used for analysis. Absolute power was then $Z$-scored relative to the mean and $\mathrm{SD}$ of the baseline. For each vehicle $(N=5)$ or oxytocin $(N=3)$ treated subject, $Z$-score values were averaged across 5 min intervals following treatment and a standard linear regression analysis with comparison of slopes was performed using GraphPad Prism (GraphPad Software).

\section{Peripheral Peptide Quantification}

To get an estimate of circulating peptide levels after intranasal treatment, we determined the peripheral plasma levels of OXT and AVP upon intranasal administration of vehicle (baseline), OXT or AVP at the highest dose tested $(1.33 \mu \mathrm{g} / \mathrm{mouse})$, using the same animal preparation and intranasal injection procedures employed for fMRI. To this aim, $0.5 \mathrm{ml}$ of blood was withdrawn from the femoral artery of male adult C57BL6/J mice, $25 \mathrm{~min}$ after intranasal administration of peptides (OXT, $n=10$; AVP $n=10$ ) or vehicle (water, $n=10$ ) in halothane anesthetized mice. Blood sampling was carried out $25 \mathrm{~min}$ after intranasal dosing to obtain an estimate of peptide levels at a time point relevant for our fMRI mapping, which covered the first 27-30 min after intranasal administration. Blood samples were centrifuged $\left(10 \mathrm{~min}, 1300 \mathrm{~g}, 4^{\circ} \mathrm{C}\right)$ and plasma samples $(0.3 \mathrm{ml})$ were kept at $-20^{\circ} \mathrm{C}$ until the radioimmunoassay procedure. OXT and AVP levels were estimated in plasma samples by using a highly sensitive and specific radioimmunoassay (RIAgnosis, Munich) as previously described (Ludwig et al, 2005; Neumann et al, 2013). Using this assay, the detection limit for OXT and AVP is in the $0.5 \mathrm{pg} / \mathrm{sample}$ range and the cross-reactivity of both neuropeptides is extremely reduced $(<0.7 \%)$. All the plasma samples were treated identically and assayed at the same time to avoid any interassay variability. To estimate the total peripheral circulating amounts of OXT and AVP, we assumed a total blood volume of $72 \mathrm{ml} / \mathrm{kg}$, corresponding to $2.16 \mathrm{ml}$ of blood in a $30 \mathrm{~g}$ male mouse (Diehl et al, 2001).

\section{RESULTS}

\section{Intranasal OXT Elicits a Composite Pattern of fMRI Activation}

Acute administration of a behaviorally active dose of OXT $(1.3 \mu \mathrm{g} / \mathrm{mouse}$, Huang et al, 2014) elicited rapid and robust rCBV increases across a widespread network of cortical and subcortical substrates (Figure $1, Z>2, p c=0.01$ ). Based on the dynamics of the functional activity elicited, the activation pattern could be segregated into two main components. A transient $(\sim 10 \mathrm{~min})$ functional activation was detected in widespread cortical and subcortical substrates, including limbic parietal and perihippocampal cortices, ventral hippocampus, and dorsal striatum. A more focal set of brain regions that included basal forebrain nuclei of the extended amygdala, piriform and insular cortex, dorsal hippocampus, thalamus, paraventricular and amygdaloid nuclei, hypothalamus, and the cerebellum showed a sustained $\mathrm{rCBV}$ increase that lasted throughout the time window covered in our experiments. This latter set of regions includes key substrates of the rodent social brain characterized by high OXT-R density such as the basal forebrain, periventricular piriform and olfactory regions, and dorsal hippocampal areas (Insel, 2010; Hammock and Levitt, 2013).

In order to explore the dose-dependence of the functional effects mapped, we repeated the functional mapping using a lower amount of OXT $(0.3 \mu \mathrm{g} /$ mouse, Supplementary Figure $\mathrm{S} 2 ; Z>2, \mathrm{pc}=0.01$ ). The dose tested is behaviorally active and elicits acute prosocial effects in adult male mice (Huang et al, 2014). The obtained pattern of rCBV activation closely replicated the one observed with higher OXT dosing, including the presence of two neuroanatomically segregable components characterized by a prolonged or more transient dynamic. These results suggest that the different regional dynamics produced by OXT administration was doseinvariant, a notion corroborated by VOI-based comparisons of the magnitude of $\mathrm{rCBV}$ response elicited by the two OXT doses tested in the transient and sustained phase (Supplementary Figure S3).

OXT administration did not produce any appreciable alterations in arterial blood pressure at both the doses tested (Supplementary Figure S4a), arguing against a peripheral cardiovascular contamination of the hemodynamic effects mapped. To corroborate a neural origin of the functional changes, we also performed LFP mapping in the dorsal hippocampus (CA1) upon intranasal administration of OXT $(1.3 \mu \mathrm{g})$ under the same anesthetic conditions of the imaging studies. We chose the dorsal hippocampus because it is a region rich in OXT receptors (Hammock and Levitt, 2013) and a key component of the network of regions exhibiting prolonged activation upon OXT administration. A robust multi-band (beta-gamma) increase in LFP power was observed in OXT-treated mice, together with a reduced delta power (Figure 2), a hallmark neurophysiological signature of neutrally driven hemodynamic responses (Logothetis, 2008). These findings suggest that intranasal OXT can rapidly modulate neural activity in deep parenchymal regions.

\section{Selective OXT Receptor Agonism Recapitulates the Activation Pattern Elicited by OXT}

In addition to binding with high affinity to OXT receptors (OXT-R), OXT display lower (but significant) affinity at vasopressin V1a and V1b receptors as well (Supplementary Figure S1). In order to probe the contribution of OXT-R in the pattern of functional activation produced by OXT, we mapped the fMRI response to intranasal administration of TGOT $(1.3 \mu \mathrm{g} / \mathrm{mouse})$, a neuropeptide OXT derivative characterized by high OXT-R affinity and selectivity over V1a and V1b vasopressin receptors (Supplementary Figure S1). fMRI mapping of intranasal TGOT revealed a functional activation pattern recapitulating key features observed with OXT, including the presence of sustained activation of basal forebrain, piriform regions, and dorsal hippocampus and transient cortical and ventral hippocampal activation (Figure 3, $\mathrm{Z}>1.7, \mathrm{pc}=0.01$ ). When quantified in volumes of interest, the transient $\mathrm{rCBV}$ response elicited by TGOT appeared to be lower than that observed with the same amount of OXT in a number of brain regions (ie, prefrontal 
a

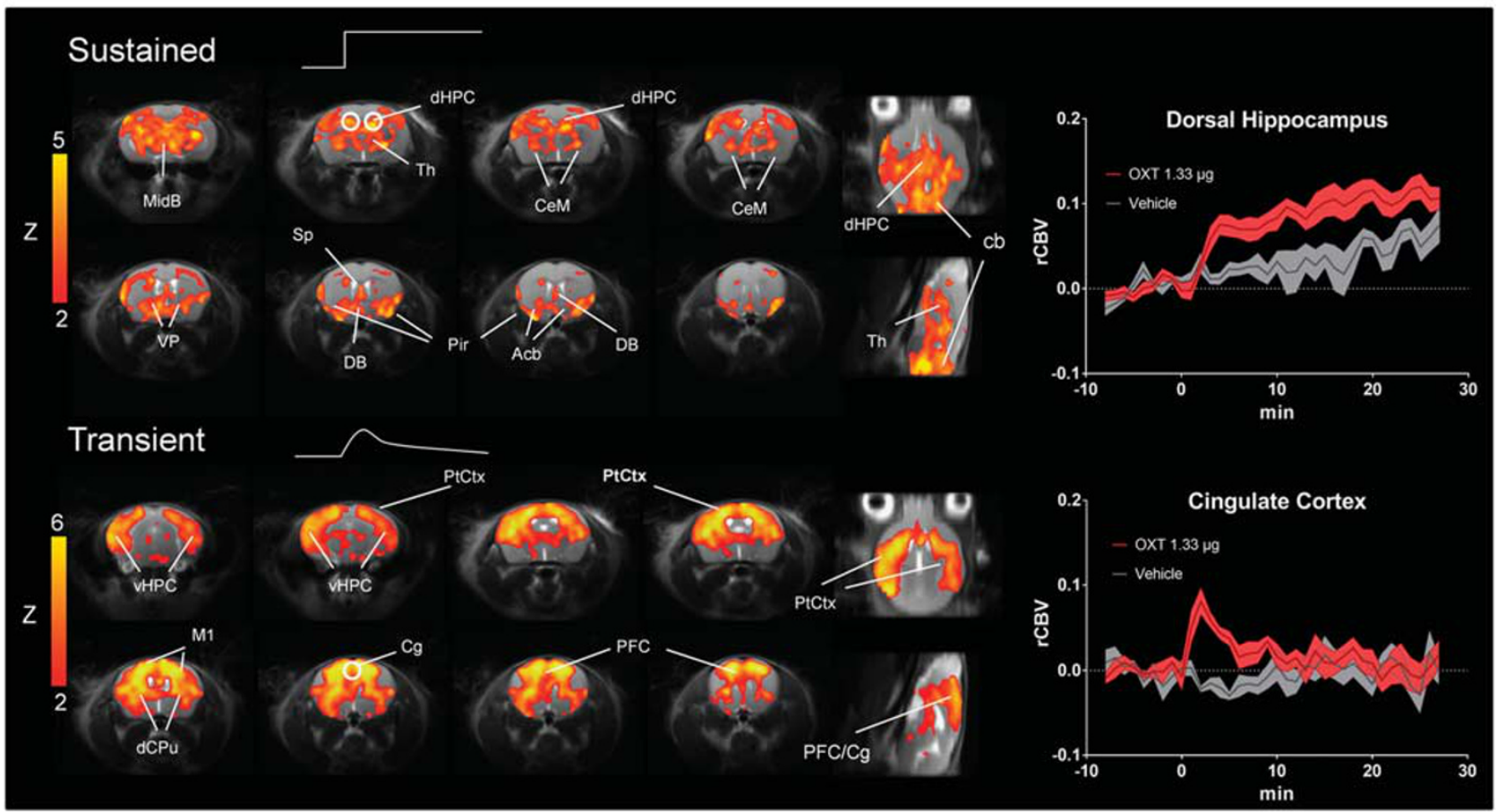

b
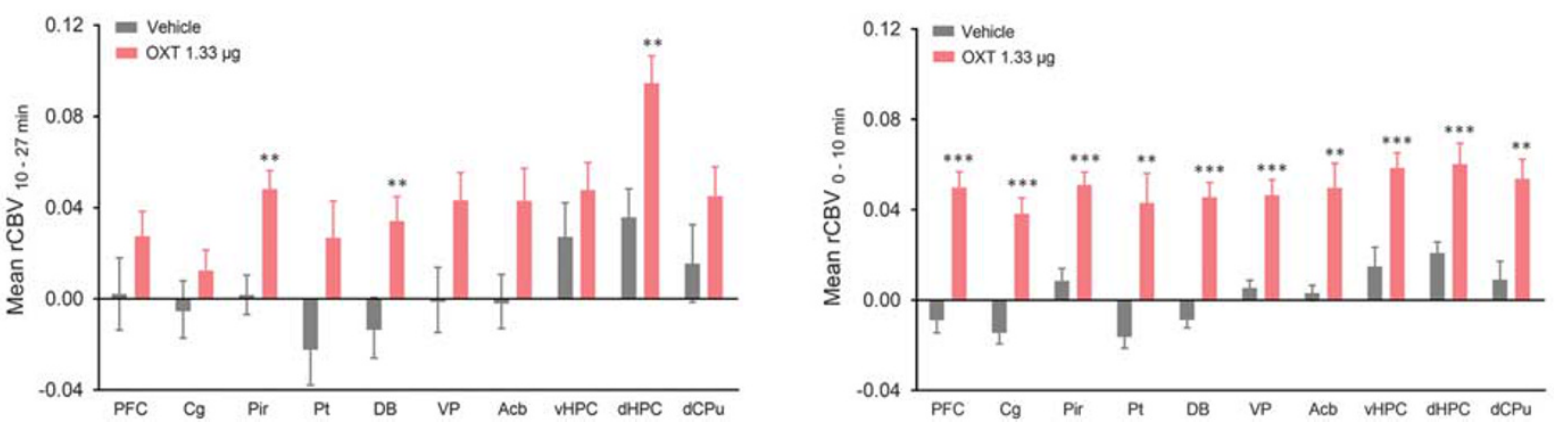

Figure I fMRI activation produced by intranasal OXT administration in the mouse brain. We mapped the functional activation produced by acute intranasal OXT in the mouse ( $1.33 \mathrm{\mu g} /$ mouse) using two different regression functions characterized by a sustained (a, top) and transient dynamics (b, bottom). The regressor functions used for fMRI mapping are depicted on top of each panel. Illustrative fMRI time courses in the representative regions of interest (identified by a circle in the activation maps) are also reported. OXT or vehicle were administered at time 0 . Data are plotted as mean \pm SEM within each group. (b) Mean rCBV response elicited by OXT as quantified in the representative volumes of interest (left: sustained, right: transient; $* * p<0.0$ I; $* * * *<0.0$ I, Student's t-test, followed by Benjamini-Hochberg correction). Acb, nucleus accumbens; cb, cerebellum; Cg, cingulate cortex; DB, diagonal band; dCPu, dorsal portion of the caudate putamen; dHPC, dorsal hippocampus; MI, primary motor cortex; PtCtx, parietal cortex; PFC, prefrontal cortex; Pir, piriform cortex; rCBV, relative cerebral blood volume; Sp, septum; Th, thalamus; VHPC, ventral hippocampus; VP, ventral pallidum.

cortex, diagonal band, ventral pallidum, ventral hippocampus, and striatum, $p<0.05$, Supplementary Figure S2), while no significant differences were observed in the same regions during the sustained activation phase (Figure 3). These results suggest a predominant contribution of OXT-R in mediating the brainwide functional effects we mapped with this neuropeptide. Similar to OXT, TGOT administration did not result in significant arterial blood pressure alterations (Supplementary Figure S4b).

\section{Intranasal AVP Produces Regional Deactivation}

We next used fMRI to map the functional effect of intranasal AVP $(1.3 \mu \mathrm{g} / \mathrm{mouse})$. Interestingly, acute intranasal administration of AVP produced rapid and widespread $\mathrm{rCBV}$ decreases (ie, deactivation) in a number of cortical and subcortical regions (Figure $4, Z>2, \mathrm{pc}=0.01$ ). The most prominent effects were observed in the medial prefrontal and infralimbic cortex, caudate putamen, parietal cortical areas, and thalamus. Foci of functional deactivation were also observed in the nucleus accumbens. The effect was rapid and sustained, with no apparent regional differences in the functional dynamics of the deactivation (Supplementary Figure S5). AVP administration was associated with a robust increase in mean arterial blood pressure (Supplementary Figure S4c), a robust correlate of central and peripheral AVP administration (Saladin and Bruni, 1993). A cardiovascular origin of the deactivation mapped with fMRI is, however, highly unlikely, because hypertensive responses exceeding the autoregulation window have been previously shown to 


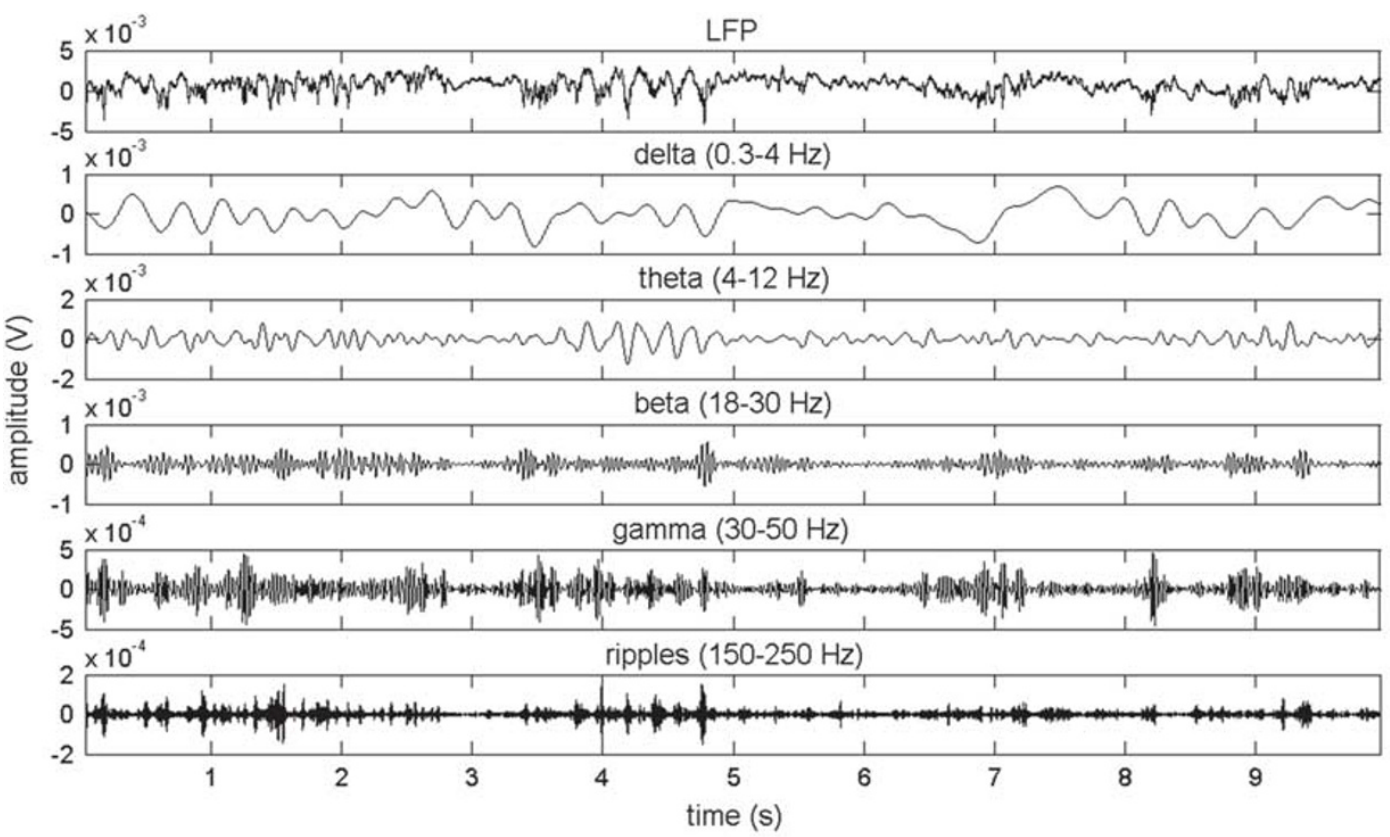

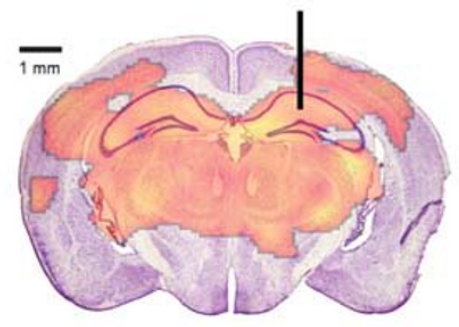

c

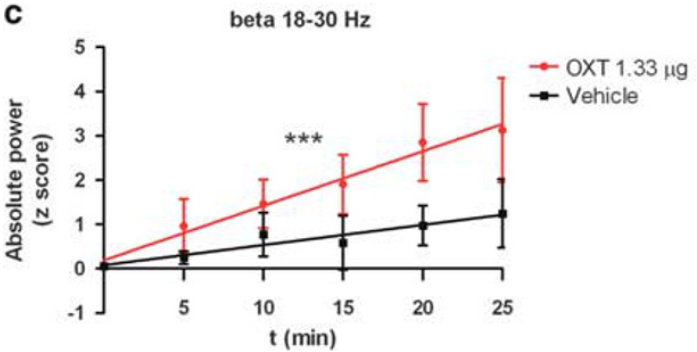

e

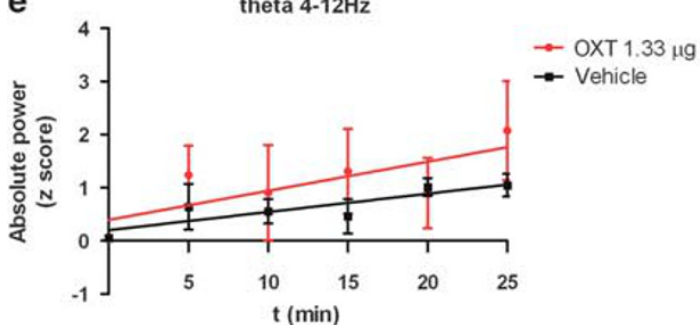

b

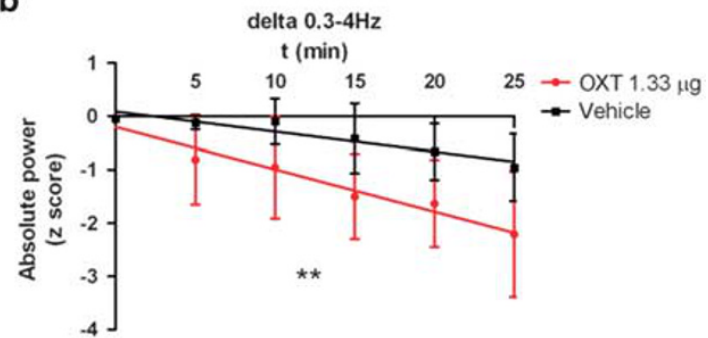

d
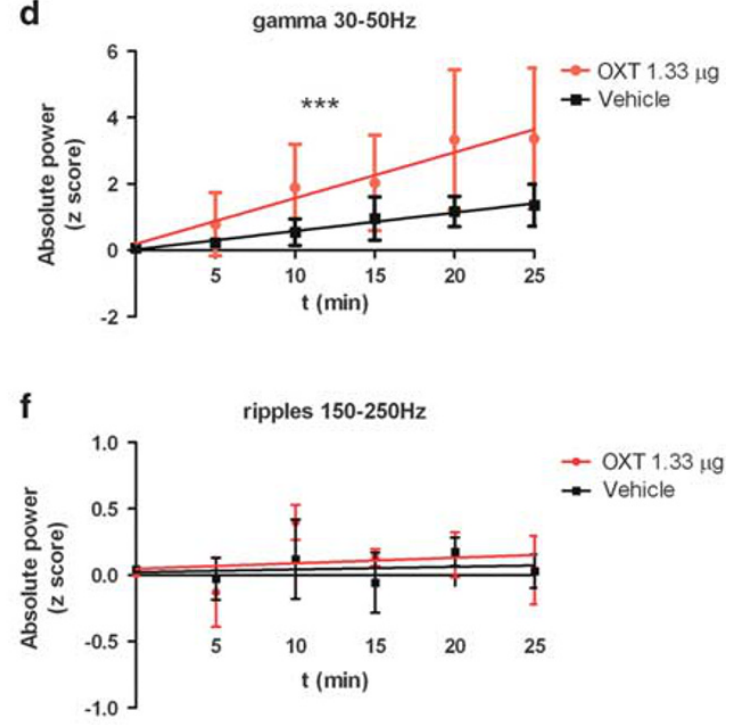

Figure 2 Intranasal OXT induced multi-band LFP response in the dorsal hippocampus. Top: Representative LFP traces recorded 20 min after OXT administration $(1.33 \mu \mathrm{g})$ in the dorsal hippocampus. (a) Representative coronal brain section with superimposed the fMRI response to OXT stimulation. The insertion path of the tungsten electrode used for hippocampal oscillation recordings is indicated by the vertical black line. (b-e) Time course of absolute power (Z-score) for delta (b), beta (c), gamma (d), theta (e), and ripple ( $f$ frequency bands upon oxytocin or vehicle application. Bars indicate the SEM of individual data points. Linear regressions (solid lines) show a significant difference in absolute power change across the first 25 min for beta, delta, and gamma oscillations (** $p \leqslant 0.01, * * * * 0.00 I)$. 

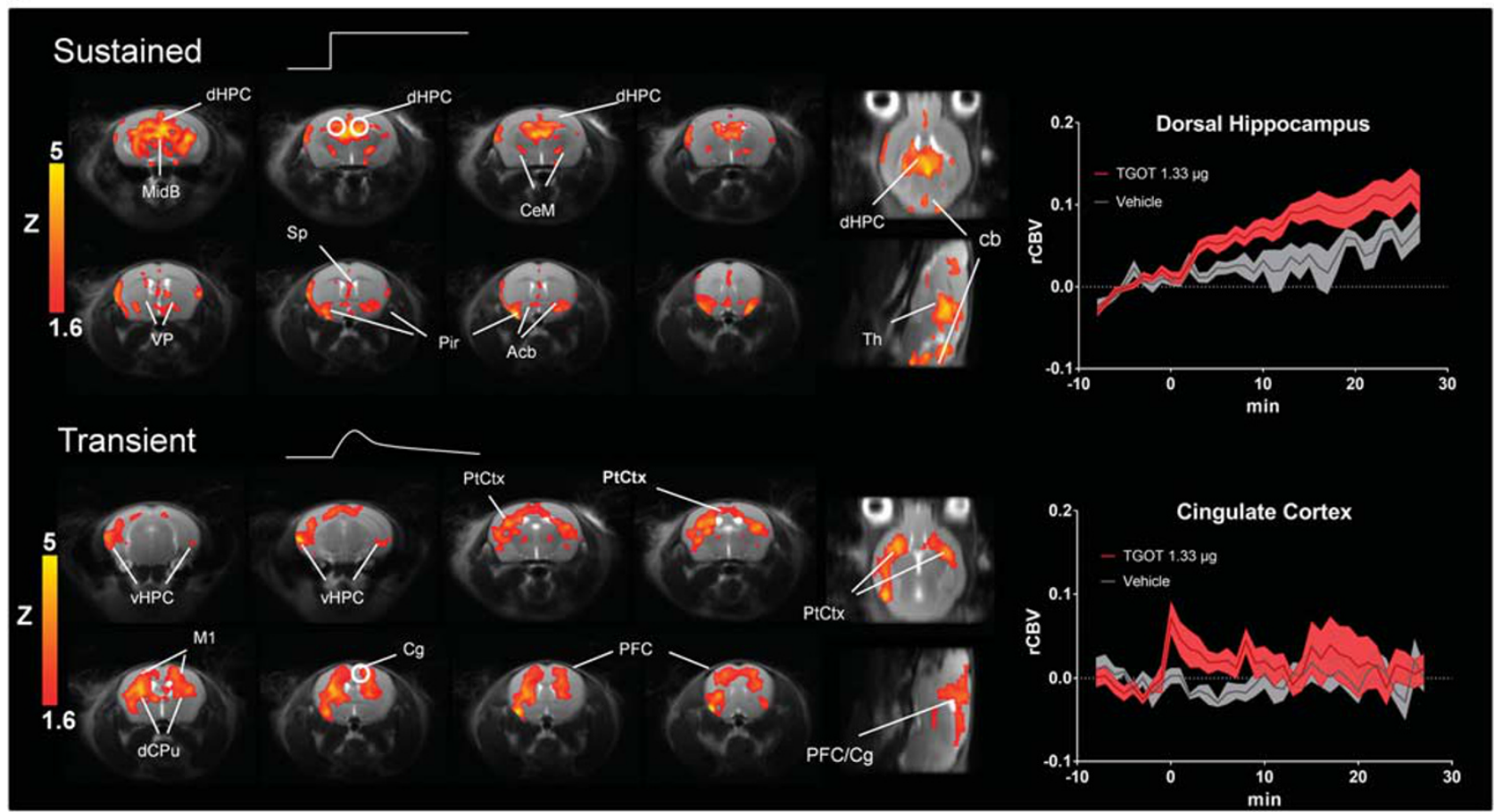

b

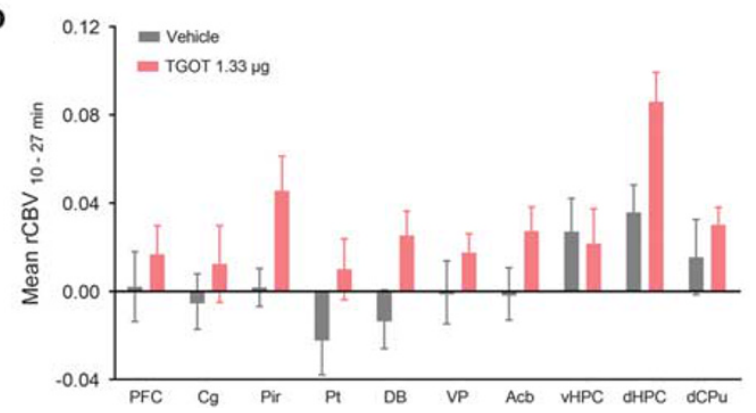

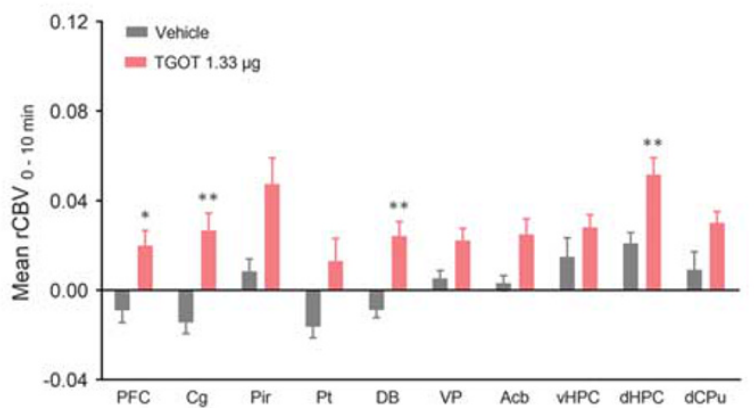

Figure $3 \mathrm{fMRI}$ activation produced by the selective OXT-R agonist TGOT in the mouse brain. As with OXT, we mapped the functional activation produced by acute intranasal TGOT areas ( $1.33 \mu \mathrm{g} / \mathrm{mouse}$ ) using two different regression functions characterized by a sustained (a, top) and transient dynamics (b, bottom). The regressor functions used for $\mathrm{MMRI}$ mapping are depicted on top of each panel. Illustrative fMRI time courses in the representative regions of interest (identified by a circle in the activation maps) are also reported. TGOT or vehicle were administered at time 0 . Data are plotted as mean \pm SEM within each group. (b) Mean rCBV response elicited by TGOT as quantified in the representative regions of interest (* $p<0.05$; $* * *<0.01$, Student's t-test, followed by Benjamini-Hochberg correction). Acb, nucleus accumbens; cb, cerebellum; Cg, cingulate cortex; DB, diagonal band; dCPu, dorsal portion of the caudate putamen; dHPC, dorsal hippocampus; Pt, parietal cortex; PFC, prefrontal cortex; Pir, piriform cortex; rCBV, relative cerebral blood volume; vHPC, ventral hippocampus; VP, ventral pallidum.

produce increased rCBV mimicking functional activation (Zaharchuk et al, 1999; Gozzi et al, 2007), as opposed to deactivation. In light of this, it is conceivable that hypertensive states exceeding autoregulation threshold could indeed have attenuated (rather than the origin of) the deactivation mapped. A perivascular effect of AVP is also similarly unlikely, because previous investigations fail to reveal Vla mRNA in parenchymal brain vasculature (Ostrowski et al, 1994).

\section{AVP-Induced Functional Deactivation Does Not Require OXT-R}

We finally investigated the receptor determinants necessary to the functional effects mapped with AVP. AVP displays significant affinity at both OXT-R and AVP (V1a and V1b) receptors (Supplementary Figure S1). Given the lack of synthetic AVP analogues devoid of OXT-R affinity, to probe the role of AVP (V1a and V1B) receptors, we administered AVP intranasally to OXT-R null mice $\left(\mathrm{Oxtr}^{-/-}\right)$. Intranasal administration of AVP in OXT-R null mice produced a pattern of deactivation in excellent agreement with that observed in control mice (Figure $4, Z>2, p c=0.01$ ). This results suggests that OXT-R are not necessary to the deactivating effect of AVP. The magnitude of AVP-induced blood pressure changes in control and OXT-R null mice were comparable (Supplementary Figure S4c).

\section{Plasma Peptide Measurements and Intravenous fMRI Mapping}

To rule out an unspecific peripheral origin of the fMRI changes mapped, we first titrated the peripheral circulating amount of peptides upon intranasal administration of OXT and AVP at the highest dose tested (Figure 5). We found that 
a

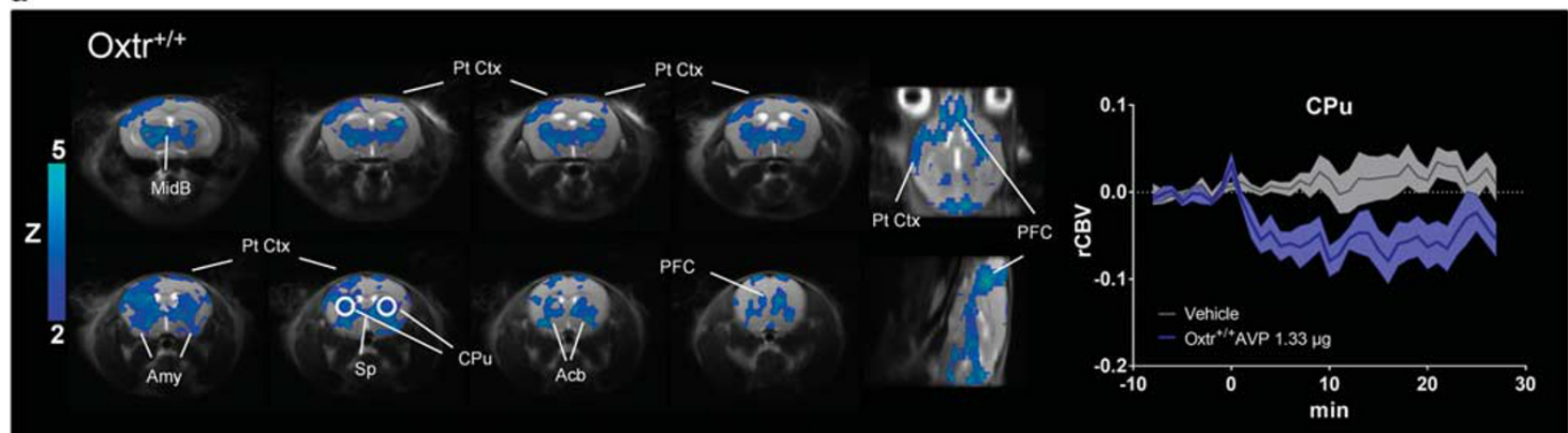

\section{Oxtr-1-}
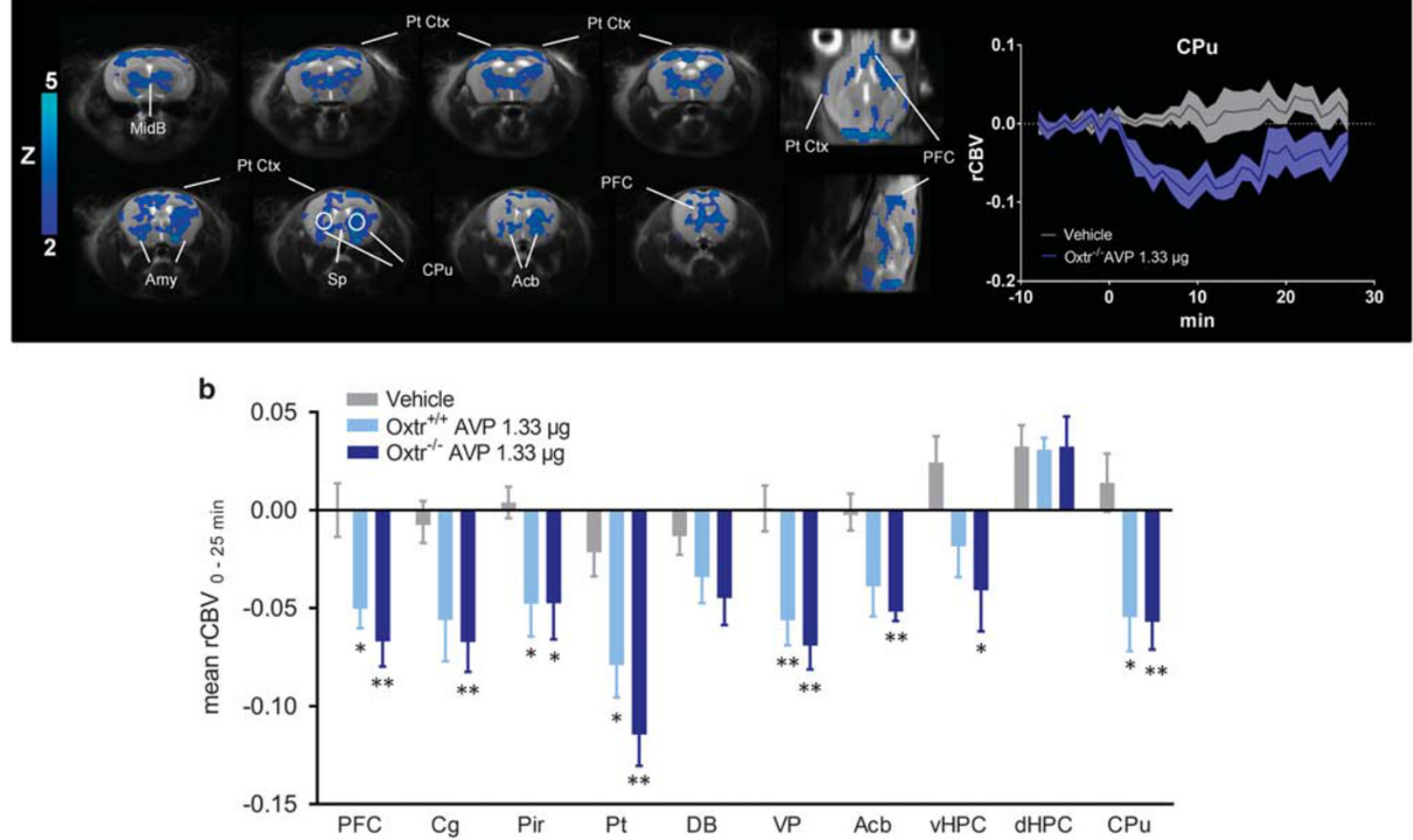

Figure 4 fMRI deactivation produced by acute intranasal administration of AVP in the mouse brain. (a) AVP-induced fMRI response was mapped both in control Oxtr ${ }^{+/+}$and Oxtr ${ }^{-1-}$ mice ( $1.33 \mu \mathrm{g} /$ mouse). The $\mathrm{fMRI}$ response was mapped with respect to vehicle-treated baseline (water) using a boxcar function, as we did not observe apparent regional differences in temporal fMRI dynamics (Supplementary Figure S5). An illustrative fMRI time course in a representative region of interest (identified by a circle in the activation maps) is reported. AVP or vehicle were administered at time 0 . (b) Mean rCBV response elicited by AVP as quantified in the representative regions of interest as a function of genotype $(* p<0.05$; ** $p<0.0$ I, Student's $t$-test, followed by Benjamini-Hochberg correction). Acb, nucleus accumbens; Amy, amygdala; Cg, cingulate cortex; DB, diagonal band; dCPu, dorsal portion of the caudate putamen; dHPC, dorsal hippocampus; MidB, midbrain; Pt, parietal cortex; PFC, prefrontal cortex; Pir, piriform cortex; Pt Ctx, parietal cortex; Sp, septum; rCBV, relative cerebral blood volume; vHPC, ventral hippocampus; VP, ventral pallidum.

the average total amount of peripherally circulating OXT and AVP $(39.8 \pm 33.2$ and $99.1 \pm 55.0 \mathrm{pg} / \mathrm{mouse}$ for OXT and AVP, respectively, assuming $2.16 \mathrm{ml}$ of total circulating blood volume; Diehl et al, 2001) was only a marginal fraction of the intranasal dose, corresponding to 0.003 and $0.007 \%$ of the total intranasal amount administered (OXT and AVP, respectively, 25 min post-administration, Figure $5 \mathrm{a}$ and b). This small amount was, however, significantly higher than what was measured under baseline conditions in vehicletreated animals (average increase was $\sim 19$-fold and $\sim 22$-fold for OXT and AVP, respectively, $p<0.01$, Student's $t$-test, Figure $5 \mathrm{a}$ and $\mathrm{b}$ ).
To rule out a contribution of the small fraction of peripherally circulating OXT and AVP to the fMRI effects mapped, we next measured the fMRI response produced by intravenous administration of OXT $(0.03 \mu \mathrm{g} /$ mouse $)$ and AVP $(0.003 \mu \mathrm{g} /$ mouse $)$ at two doses greatly exceeding the quantified peripheral levels $(\sim 750$ - and $\sim 30$-fold the mean peripheral OXT and AVP levels measured, respectively) so as to account for possible intersubject variability in vascular leakage of these peptides. We reasoned that, if such large doses did not recapitulate the observed intranasal fMRI patterns, a peripheral origin of our functional maps would be highly unlikely. AVP was tested at the highest dose ensuring 
a

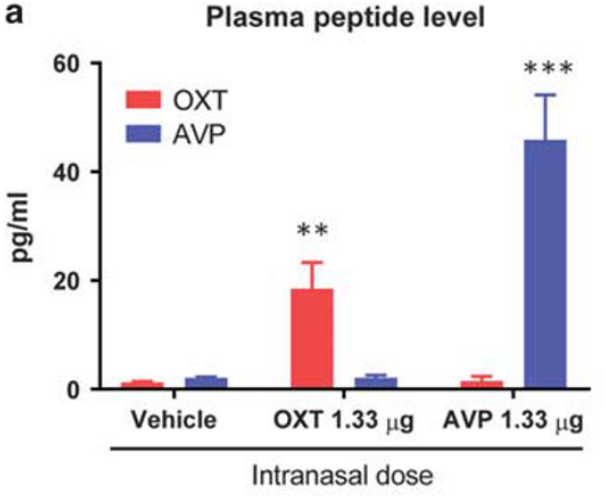

b

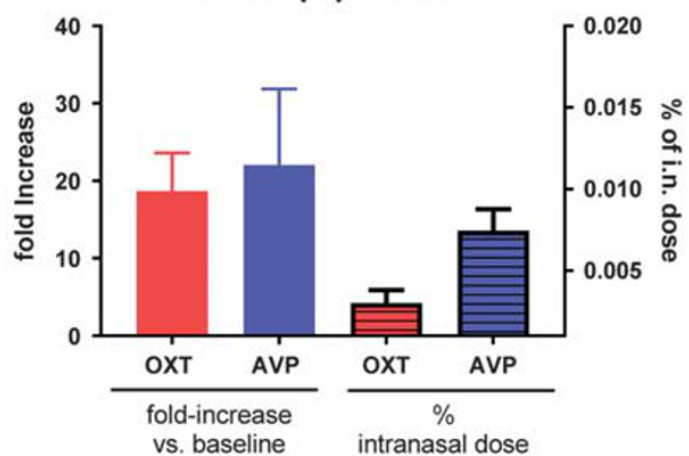

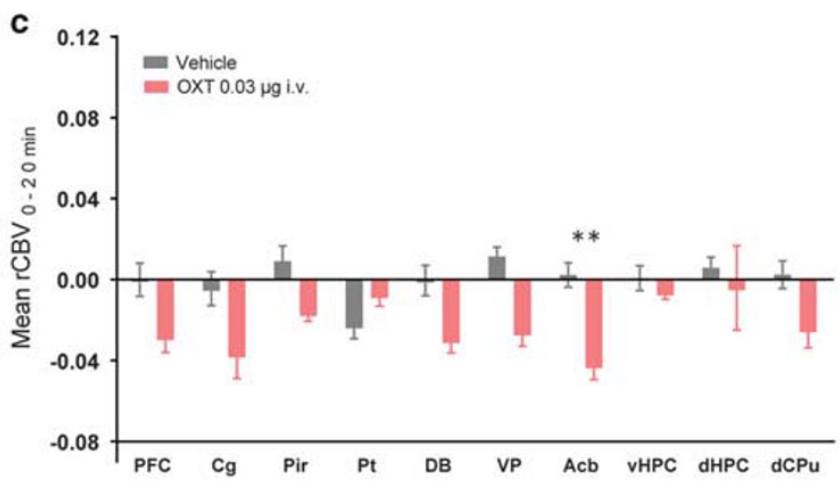

d

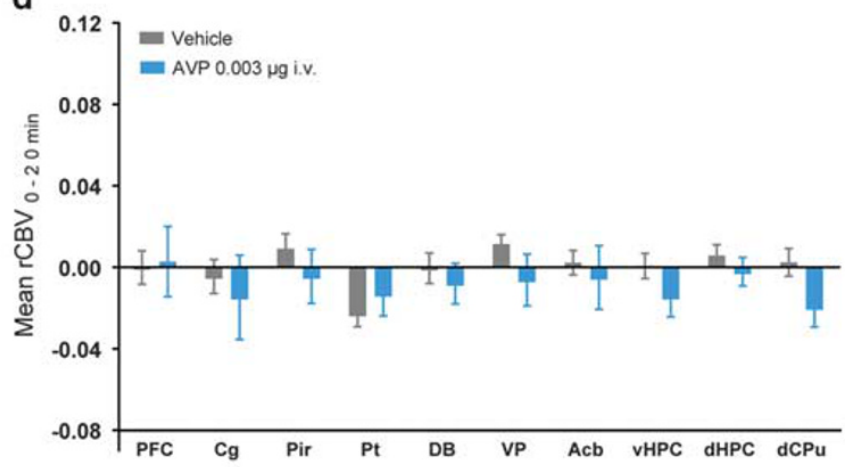

Figure 5 Plasma peptide measurements and intravenous fMRI mapping. (a) Plasma OXT and AVP levels (pg/ml) 25 min after intranasal oxytocin or

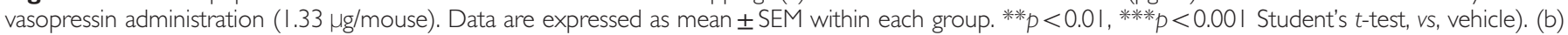
Plasma OXT and AVP levels expressed as fold increase compared with mean baseline levels (left panel) or as percentage with respect to the administered intranasal dose (right panel). (c) Mean rCBV response elicited by intravenous administration of OXT (0.03 $\mu g /$ mouse) as quantified in representative volumes of interest. The response to OXT was significantly reduced compared with vehicle only in the nucleus accumbens (Acb) VOls (*** $p<0.0 \mathrm{I}$, Student's $t$-test, followed by Benjamini-Hochberg correction). (d) Mean rCBV response elicited by intravenous administration of AVP (0.003 $\mu g / m o u s e)$ as quantified in the representative volumes of interest. Data are plotted as mean \pm SEM within each group. Acb, nucleus accumbens; Cg, cingulate cortex; DB, diagonal band; $\mathrm{dCPu}$, dorsal portion of the caudate putamen; dHPC, dorsal hippocampus; Pt, parietal cortex; PFC, prefrontal cortex; Pir, piriform cortex; rCBV, relative cerebral blood volume; VHPC, ventral hippocampus; VP, ventral pallidum.

a peripheral response not exceeding cerebral blood flow autoregulation in individual animals. Notably, these studies failed to replicate the fMRI response obtained upon intranasal administration (Figure $5 \mathrm{c}$ and d. Supplementary Figures S6 and S7). Specifically, AVP did not reveal any significant fMRI response with respect to intravenous saline injection, while OXT produced a trend for a weak deactivation (as opposed to the activation observed intranasally) in some brain regions, an effect that reached statistical significance in the nucleus accumbens (Figure 5, $p<0.01$ ). The contrasting direction of effect produced by peripheral OXT administration despite the use of OXT amounts largely exceeding the peptide levels quantified in plasma argue against a contribution of circulating OXT to the fMRI response mapped with intranasal peptide administration, thus corroborating a central origin of the intranasal fMRI signals. In keeping with this notion, intravenous administration of TGOT failed to elicit significant alterations in any of the brain regions examined (Supplementary Figures S6 and S7), although as seen with OXT, a trend for a reduction in $\mathrm{rCBV}$ was observed in some brain area multiple regions. Intravenous administration of OXT and TGOT produced small and transient blood pressure increases, while AVP induced a sustained blood pressure increase reminiscent of that observed upon intranasal administration (Supplementary Figure S8).

\section{DISCUSSION}

We used phMRI to map the large-scale functional substrates modulated by acute intranasal administration of OXT and AVP in the mouse brain and demonstrate that both neuropeptides elicit a rapid and sustained modulation of parenchymal and periventricular brain substrates, including key regions involved in social and emotional behavior. Importantly, we document an unexpected opposing modulatory influence of OXT and AVP on brainwide functional substrates and demonstrate that this effect is not recapitulated by intravenous peptide administration, thus corroborating a central origin of the observed fMRI changes. From a purely methodological standpoint, our study represents the first demonstration of the possibility to non-invasively map the substrates recruited by intranasally administered peptides in mice, thus paving the way to the use of this approach to the characterization of CNS effects of centrally active biologicals that do not cross the blood-brain barrier.

The pattern of sustained fMRI activation obtained with OXT encompasses limbic brain substrates previously 
described to be modulated by this neuropeptide in humans. In particular, a recent study from Paloyelis et al (2014) permits to directly compare our results with the fMRI response produced by OXT under task-free conditions in men. In agreement with our findings, human cerebral blood flow mapping of OXT revealed the sustained activation of an extended network of regions, including the hippocampus, amygdala, ventral striatum, basal forebrain areas, anterior insula, midbrain brain stem nuclei, and cerebellum, corroborating the notion of a cross-species conservation of the substrates modulated by this neuropeptide (Insel, 2010) and the translational relevance of our approach. The presence of foci of fMRI activation in central amygdaloid nuclei is also consistent with human task-based fMRI mapping indicating this region as a key substrate of the emotional and affective properties of OXT (Kirsch et al, 2005; Meyer-Lindenberg et al, 2011), although in our maps the amygdala did not appear to be activated in all its subcomponents. In contrast with human fMRI mapping (Labuschagne et al, 2010; Sripada et al, 2013; Paloyelis et al, 2014; Riem et al, 2014), we did not observe sustained activation of cingulate and prefrontal areas, although these regions exhibited a robust transient activation within the first $10-15 \mathrm{~min}$ after OXT administration. Because human OXT investigations typically cover late injection time points (typically $>30 \mathrm{~min}$ after administration), it is not clear whether such rapid and transient response reflects the intrinsic dynamics of intranasal route or whether it is the consequence of cross-species or experimental discrepancies.

Notably, the distribution of the sustained fMRI response to OXT exhibits significant overlap with the limbic brain substrates characterized by OXT-R expression in the rodent brain, a correspondence that appears to be especially prominent in rostral brain regions (Dumais et al, 2013; Freeman and Young, 2016) and that empirically supports a central origin for the effects mapped. We also observed robust hippocampal activation in line with the key contribution of this neuropeptide as a facilitator of information processing in the brain (Owen et al, 2013; ShamayTsoory and Abu-Akel, 2016) and the high OXT-R abundance in this region. The observation of qualitatively similar patterns of rCBV activation upon OXT and TGOT administration corroborates a predominant contribution of the OXT-R in mediating these functional signals, although this finding alone does not permit to completely rule out a nonnegligible regional involvement of $\mathrm{Vla}$ or $\mathrm{V} 1 \mathrm{~b}$ receptors in the fMRI response mapped. Further studies using selective pharmacological manipulations in genetic modified animals are required to fully disambiguate this contribution, such as OXT dosing in OXT-KO mice or pharmacological manipulation with selective OXT or AVP receptor antagonists. The lower functional response observed with TGOT and lack of efficacy at the lower dose tested were unexpected, given the higher receptor affinity of this peptide at OXT-R (Busnelli et al, 2013) and its greater in vivo potency upon intraventricular administration (Sala et al, 2013). Although the origin of this discrepancy remain unclear, differences in the intranasal pharmacodynamics, stability, or biodistribution of these two analogous, but structurally distinct, peptides could possibly account for this unexpected finding.

The sustained pattern of OXT activation we observed is also in excellent agreement with previous fMRI mapping of this neuropeptide upon central intraventricular administration in the rat (Febo et al, 2005; Ferris et al, 2015), a finding that underscores the efficiency of the intranasal route in enabling CNS access to bulky neuroactive peptides. The origin and significance of the different temporal response observed in cortical and subcortical regions are, however, unclear. A time-dependent involvement of different downstream neurotransmitter systems affected by OXT may underlie this observation. For example, the central nucleus of the amygdala, a key substrate of OXT action, can directly modulate central cholinergic function (Gozzi et al, 2010) and the transient cortical involvement observed with OXT (and TGOT) is reminiscent of the widespread functional activation obtained upon endogenous cholinergic activation (Gozzi et al, 2010). Interestingly, evidence of high OXT-R density in cholinergic nuclei has been recently described in primates (Freeman and Young, 2016). A transient disinhibition of cholinergic (Boccia and Baratti, 2000) or serotonergic activity (Dolen et al, 2013) may underscore the observed transient functional response. Further studies involving the selective manipulation of neurotransmitter systems involved in OXT function are required to probe the validity of this hypothesis.

Three main functional pathways into the cranial cavity have been proposed for intranasally delivered neuropeptides (Veening and Olivier, 2013): (a) intra-axonal and transneuronal transport via the olfactory pathways; (b) via perineuronal and other spaces along the olfactory fibers and cranial nerves to enter the arachnoid space and cerebrospinal fluid (CSF); and (c) via the peripheral blood stream and the blood-brain barrier (BBB) after crossing the mucosal walls of the nasal cavity. Although our fMRI mapping does not permit a clear mechanistic assessment of the entry route of OXT in the cranial cavity, the lack of blood pressure alterations, a hallmark cardiovascular reaction to peripheral OXT administration (Thomas et al, 2007), together with the low BBB permeability of this neuropeptide (Mens et al, 1983) strongly argue against a contribution of peripherally circulating OXT in the functional effects mapped. A major involvement of intra-axonal and transneural transport is equally unlikely, given the low efficiency and very slow nature of this kind of translocation mechanism (Dhuria et al, 2010). A significant contribution of extra-axonal and perineuronal volume transmission via CSF is the most probably explanation for the fast and distributed functional rCBV response observed in our study, a hypothesis consistent with in vivo microdialysis measurements in the rodent brain upon intranasal administration (Neumann et al, 2013). A detailed discussion of the mechanistic and neuroanatomical foundations of this putative nose-to-brain entry route has been recently summarized by Veening and Olivier (2013).

As with OXT, the fMRI pattern of AVP deactivation includes brain substrates (cingulate, temporal-parietal cortex, caudate nuclei, and amygdala) recently described to be modulated by this neuropeptide in task-based fMRI mapping in humans (Zink and Meyer-Lindenberg, 2012). However, the lack of task-free intranasal fMRI mapping of AVP in humans does not permit to directly compare first-order effects of this neuropeptide across species. It is, however, interesting to note that, similar to our imaging findings, AVP was recently reported to induce brain deactivation in 
humans (Lee et al, 2013). Interestingly, not all the regions characterized by high $\mathrm{V} 1 \mathrm{a}$ or $\mathrm{V} 1 \mathrm{~b}$ receptor abundance appeared to be the modulated by AVP. For example, no functional modulation was observed in the hypothalamus, ventral diagonal band (rich in V1a receptors; Hernando et al, 2001), or the hippocampus (rich in both V1a and V1b receptors; Hernando et al, 2001; Young et al, 2006). This observation does not imply an unspecific origin of the imaging effects mapped, because hemodynamic responses are known to reflect poly-synaptic integrative processes that do not necessarily serve as a reliable proxy of local receptor distribution (Logothetis, 2008).

A comparison of our OXT doses with those utilized in analogous rodent investigations suggests that the amounts of peptide we tested are in line with, or lower than, what was previously assayed in acute intranasal studies, hence making our functional mapping relevant for rodent investigators. For example, when normalized by body weight (6.7 and $26.7 \mathrm{IU} / \mathrm{kg}$ ), our OXT doses are similar to those recently shown to produce acute prosocial effects (ie, 5 and $10 \mathrm{IU} / \mathrm{Kg}$, Huang et al, 2014) and much lower than the amount employed to induce conditioned social preference (240 IU/kg, Kosaki and Watanabe, 2016), rescue autismlike behaviors (120 IU/kg, Penagarikano et al, 2015), or investigate brain and plasma peptide biodistribution in mice (120 IU/Kg, Neumann et al, 2013). Similar amounts of OXT have been investigated in acute intranasal studies in the rat as well, eg, $40 \mathrm{IU} / \mathrm{Kg}$, by Neumann et al (2013) and Calcagnoli et al (2015). Examples of lower intranasal OXT dosing have been described in prairie voles (eg, 0.08, 0.8, and $8 \mathrm{IU} / \mathrm{Kg}$, Bales et al, 2015) and mice (Bales et al, 2014), although in such cases the peptide was administered chronically for several weeks.

If directly extrapolated to humans, our OXT doses would correspond to the acute administration of 335 and 1335 IU to a $50 \mathrm{~kg}$ human individual. Human acute dosing ranges approximately from 10 to $40 \mathrm{IU}$, with a large prevalence of a $24 \mathrm{IU} /$ dose administered once or twice daily (Davis et al, 2013; Anagnostou et al, 2014; Kirkpatrick et al, 2014). When compared with these amounts, our low OXT dose would thus be approximately 7-10-fold higher than the daily OXT dose employed by human investigators and the high dose 32-40-fold higher. However, two critical experimental factors should be considered when direct mouse to men dose extrapolation are attempted. First, human dosing employs OXT spraying/nebulization, a modality resulting in enhanced biodistribution and higher CNS drug concentrations (Henry et al, 1998; Hoekman and Ho, 2011). This aspect seems to be especially relevant for neuropeptides, such as OXT and AVP. For example, in macaques aerosolized OXT, but not intranasal OXT administration, resulted in significant increases in lumbar CSF OXT levels (Modi et al, 2014). Similarly, nebulized AVP (Ramos et al, 2014), but not intranasal deposition of the peptide (Ludwig et al, 2013), elicited behavioral responses in rats. The higher bioavailability and efficiency of nebulized administration with respect to rodent liquid administration poses a first interpretational limit to direct mouse-to-human extrapolation of OXT and AVP dosing that can account for the overall use of higher peptide amounts in rodent investigations. Similarly, direct rodent to human dose extrapolations typically neglect established interspecies differences in metabolic rate and pharmacokinetic parameters, a contribution that can be rigorously formalized in allometric dose scaling (reviewed by Sharma and McNeill, 2009). Although the validity and specific assumptions underlying allometric dose scaling for intranasal peptides remain completely unexplored, it is worth noting that FDA guidelines based on metabolism and body surface area recommend a mouseto-human dose extrapolations factor of 0.16 (USFDA, 2005; Sharma and McNeill, 2009). Hence, allometric scaling alone could plausibly account for the different absolute dosing employed in intranasal rodent studies with respect to humans. Together with well-documented neuroanatomical differences in the architecture of rodent and human turbinates (Harkema et al, 2006), these considerations highlight the need to exercise caution when directly extrapolating dose across species and provide a possible explanation of the overall larger intranasal peptide doses employed by rodent researchers. These limitations, however, are mostly quantitative and as such do not preclude careful qualitative and mechanistic extrapolations of rodent pharmacological and functional findings to human OXT and AVP investigations.

Peripheral cardiovascular changes can affect both BOLD and CBV-weighted (Kalisch et al, 2001; Luo et al, 2003; Gozzi et al, 2007) fMRI responses. The use of rCBV-weighted mapping has, however, the advantage of permitting an unambiguous assessment of the direction and contribution of arterial blood pressure changes to central hemodynamic responses. Indeed, previous work from us (Gozzi et al, 2007) and others (Zaharchuk et al, 1999) showed that halothane anesthesia preserves cerebral autoregulation over a large range of blood pressure and that blood pressure values exceeding the upper autoregulation limit results in increased rCBV mapping. To our knowledge, no such systematic assessment has been described for BOLD fMRI. These findings corroborate a central origin of the $\mathrm{rCBV}$ response produced by AVP, because the peptide produced negative rCBV responses in the face of positive blood pressure increases. rCBV fMRI also has the additional advantage of being more easily interpretable and closely related to underlying neuronal activity than BOLD fMRI, for which examples of dissociation between hemodynamic parameters and neuronal signals have been described (Maier et al, 2008; Schridde et al, 2008).

The observation of opposing brainwide functional effects of AVP and OXT was unexpected, because this is at odds with the converging pharmacological profile, molecular pathways, and electrophysiological responses elicited by these two neuropeptides, which can similarly increase neuronal excitability (Raggenbass, 2001; Stoop, 2014). A divergent effect of OXT and AVP is also in contrast with the dominant view of a concerted contribution of these neuropeptides to social cognition, social memory, and affiliative behavior (Ferris, 2008; Insel, 2010), and evidence that both these neuropeptides can improve social deficits in mouse models of impaired OXT-mediated signaling (Sala et al, 2011). As discussed above, a role of peripheral cardiovascular confounds in the effect of AVP is highly unlikely. Although the use of light anesthesia could potentially affect the sign and distribution of the functional changes mapped, multiple lines of evidence converge to suggest that fMRI pattern here identified are representative of OXT and AVP substrates in 
conscious states. For example, the light anesthetic regimen employed preserves cerebral blood flow autoregulation (Gozzi et al, 2007), cortical electrical responsiveness (Orth et al, 2006; Gozzi et al, 2012), and spontaneous brainwide functional couplings (Sforazzini et al, 2014; Gozzi and Schwarz, 2015), and previous work demonstrated a negligible influence of this anesthetic on the functional activation produced by several psychoactive drugs, when compared with awake conditions (Gozzi et al, 2008; Gozzi et al, 2012).

It is conceivable that the observed functional divergence may reflect a regional modulation of inhibitory and excitatory cell populations, an effect that could be mediated by differences in the cellular and subcellular distribution of the receptor effectors of OXT and AVP. Recent studies showing that OXT can locally disinhibit cortical regions involved in maternal behavior (Marlin et al, 2015) and influence fast spiking inhibition in hippocampal neurons (Owen et al, 2013) lend empirical evidence to this hypothesis. In keeping with this, evidence that AVP can increase GABAergic neurotransmission in hypothalamic regions has also been described (Hermes et al, 2000), and microcircuital divergences in the modulatory action of these neuropeptide on specific brain substrates (ie, the amygdala) have been recently reported (Huber et al, 2005; Stoop, 2012). Notwithstanding the unclear origin of this phenomenon, the observation of functionally divergent responses to OXT and AVP is of interest, as it may serve as a possible brainwide functional substrate for some of the differential behavioral outputs previously described upon exogenous stimulation of these neuropeptide systems in rodents. Specifically, rodent studies have highlighted a differential contribution of AVP and OXT systems in affective and emotional responses, stress-coping (reviewed by Neumann and Landgraf, 2012), and motor-sensory regulation (Stoop, 2014). Although an extrapolation of our mouse data to human clinical research must be exercised with caution, the opposing functional effects mapped may also be of relevance for the therapeutic investigation of these neuropeptides in human conditions characterized by social impairments. Our data indeed suggest that the two peptides do not necessarily produce concordant brainwide modulation, at least in the healthy brain with normal endogenous levels of OXT. This observation challenges the notion of an overlapping action of exogenous AVP or OXT as facilitators of social function, a concept at the basis of clinical testing of these agents as normalizers of social impairments (Insel et al, 1999), and calls for a deeper investigation of the macroscale substrates engaged by OXT and AVP and their relevance to the putative therapeutic profile of these agents.

In conclusion, we mapped the macroscale functional substrates engaged by intranasal administration of OXT and AVP and show a previously unreported divergence in the brainwide functional effect modulated by the two neuropeptides. Our findings shed light on the dynamics and central substrates recruited by intranasal OXT and AVP. The observation of a functional divergence, rather than a concordant modulatory action, calls for a deeper investigation of the systems-level mechanisms by which exogenous OXT and AVP modulate brain function and exert their putative therapeutic effects.

\section{FUNDING AND DISCLOSURE}

A. Gozzi received funding from the Simons Foundation (SFARI 314688 and 400101). The authors declare no conflict of interest.

\section{REFERENCES}

Aguilera G, Pham Q, Rabadan C (1994). Regulation of pituitary vasopressin receptors during chronic stress: relationship to corticotroph responsiveness. J Neuroendocrinol 6: 299-304.

Anagnostou E, Soorya L, Brian J, Dupuis A, Mankad D, Smile S et al (2014). Intranasal oxytocin in the treatment of autism spectrum disorders: a review of literature and early safety and efficacy data in youth. Brain Res 1580: 188-198.

Bakermans-Kranenburg MJ, van IJzendoorn MH (2013). Sniffing around oxytocin: review and meta-analyses of trials in healthy and clinical groups with implications for pharmacotherapy. Transl Psychiatry 3: e258.

Bales KL, Solomon M, Jacob S, Crawley JN, Silverman JL, Larke RH et al (2014). Long-term exposure to intranasal oxytocin in a mouse autism model. Transl Psychiatry 4: e480.

Bales KL, Perkeybile AM, Conley OG, Lee MH, Guoynes CD, Downing GM et al (2015). Chronic intranasal oxytocin causes long-term impairments in partner preference formation in male prairie voles. Biol Psychiatry 74: 180-188.

Baumgartner T, Heinrichs M, Vonlanthen A, Fischbacher U, Fehr E (2008). Oxytocin shapes the neural circuitry of trust and trust adaptation in humans. Neuron 58: 639-650.

Boccia MM, Baratti CM (2000). Involvement of central cholinergic mechanisms in the effects of oxytocin and an oxytocin receptor antagonist on retention performance in mice. Neurobiol Learn Mem 74: 217-228.

Born J, Lange T, Kern W, McGregor GP, Bickel U, Fehm HL (2002). Sniffing neuropeptides: a transnasal approach to the human brain. Nat Neurosci 5: 514-516.

Bosch OJ, Pförtsch J, Beiderbeck DI, Landgraf R, Neumann ID (2010). Maternal behaviour is associated with vasopressin release in the medial preoptic area and bed nucleus of the stria terminalis in the rat. J Neuroendocrinol 22: 420-429.

Busnelli M, Bulgheroni E, Manning M, Kleinau G, Chini B (2013). Selective and potent agonists and antagonists for investigating the role of mouse oxytocin receptors. J Pharmacol Exp Ther 346: 318-327.

Calcagnoli F, Kreutzmann JC, de Boer SF, Althaus M, Koolhaas JM (2015). Acute and repeated intranasal oxytocin administration exerts anti-aggressive and pro-affiliative effects in male rats.. Psychoneuroendocrinology 51: 112-121.

Davis MC, Lee J, Horan WP, Clarke AD, McGee MR, Green MF et al (2013). Effects of single dose intranasal oxytocin on social cognition in schizophrenia. Schizophr Res 147: 393-397.

Dhuria SV, Hanson LR, Frey WH (2010). Intranasal delivery to the central nervous system: mechanisms and experimental considerations. J Pharm Sci 99: 1654-1673.

Diehl K-H, Hull R, Morton D, Pfister R, Rabemampianina Y, Smith $\mathrm{D}$ et al (2001). A good practice guide to the administration of substances and removal of blood, including routes and volumes. J Appl Toxicol 21: 15-23.

Dolen G, Darvishzadeh A, Huang KW, Malenka RC (2013). Social reward requires coordinated activity of nucleus accumbens oxytocin and serotonin. Nature 501: 179-184.

Donaldson ZR, Young LJ (2008). Oxytocin, vasopressin, and the neurogenetics of sociality. Science 322: 900-904.

Donnelley M, Morgan KS, Fouras A, Skinner W, Uesugi K, Yagi N et al (2009). Real-time non-invasive detection of inhalable particulates delivered into live mouse airways. J Synchrotron Radiat 16: 553-561. 
Dumais KM, Bredewold R, Mayer TE, Veenema AH (2013). Sex differences in oxytocin receptor binding in forebrain regions: correlations with social interest in brain region- and sex- specific ways. Horm Behav 64: 693-701.

Ebstein RP, Israel S, Lerer E, Uzefovsky F, Shalev I, Gritsenko I et al (2009). Arginine vasopressin and oxytocin modulate human social behavior. Ann NY Acad Sci 1167: 87-102.

Errico F, D'Argenio V, Sforazzini F, Iasevoli F, Squillace M, Guerri $G$ et al (2015). A role for D-aspartate oxidase in schizophrenia and in schizophrenia-related symptoms induced by phencyclidine in mice. Transl Psychiatry 5: e512.

Febo M, Numan M, Ferris CF (2005). Functional magnetic resonance imaging shows oxytocin activates brain regions associated with mother-pup bonding during suckling. J Neurosci 25: 11637-11644.

Feifel D, Shilling PD, Macdonald K (2016). A review of oxytocin's effects on the positive, negative, and cognitive domains of schizophrenia. Biol Psychiatry 79: 222-233.

Ferrari L, Turrini G, Crestan V, Bertani S, Cristofori P, Bifone A et al (2012). A robust experimental protocol for pharmacological fMRI in rats and mice. J Neurosci Methods 204: 9-18.

Ferris CF (2008). Functional magnetic resonance imaging and the neurobiology of vasopressin and oxytocin. Prog Brain Res 170: 305-320.

Ferris CF, Yee J, Kenkel W, Dumais KM, Moore K, Veenema AH et al (2015). Distinct BOLD activation profiles following central and peripheral oxytocin administration in awake rats. Front Behav Neurosci 9: 245.

Freeman SM, Young LJ (2016). Comparative perspectives on oxytocin and vasopressin receptor research in rodents and primates: Translational implications. J Neuroendocrinol 28: 1-12.

Gozzi A, Schwarz AJ (2015). Large-scale functional connectivity networks in the rodent brain. Neuroimage 127: 496-509.

Gozzi A, Schwarz AJ, Reese T, Crestan V, Bifone A (2008). Drug-anaesthetic interaction in phMRI: the case of the pyschotomimetic agent phencyclidine. Magn Reson Imaging 26: 999-1006.

Gozzi A, Ceolin L, Schwarz A, Reese T, Bertani S, Crestan V et al (2007). A multimodality investigation of cerebral hemodynamics and autoregulation in pharmacological MRI. Magn Reson Imaging 25: 826-833.

Gozzi A, Colavito V, Seke Etet PF, Montanari D, Fiorini S, Tambalo $S$ et al (2012). Modulation of fronto-cortical activity by modafinil: a functional imaging and fos study in the rat. Neuropsychopharmacology 37: 822-837.

Gozzi A, Apar J, Giovanelli A, Bertollini C, Crestan V, Schwarz AJ et al (2010). A neural switch for active and passive fear. Neuron 67: 656-666.

Guastella AJ, Hickie IB (2016). Oxytocin treatment, circuitry and autism: a critical review of the literature placing oxytocin into the autism context. Biol Psychiatry 79: 234-242.

Hammock E, Levitt P (2013). Oxytocin receptor ligand binding in embryonic tissue and postnatal brain development of the C57BL/ $6 \mathrm{~J}$ mouse. Front Behav Neurosci 7: 195.

Harkema JR, Carey SA, Wagner JG (2006). The nose revisited: a brief review of the comparative structure, function, and toxicologic pathology of the nasal epithelium. Toxicol Pathol 34: 252-269.

Henry RJ, Ruano N, Casto D, Wolf RH (1998). A pharmacokinetic study of midazolam in dogs: nasal drop vs. atomizer administration. Pediatr Dent 20: 321-326.

Hermes MLHJ, Ruijter JM, Klop A, Buijs RM, Renaud LP (2000). Vasopressin increases GABAergic inhibition of rat hypothalamic paraventricular nucleus neurons in vitro. J Neurophysiol 83: 705-711.

Hernando F, Schoots O, Lolait SJ, Burbach JP (2001). Immunohistochemical localization of the vasopressin $\mathrm{V} 1 \mathrm{~b}$ receptor in the rat brain and pituitary gland: anatomical support for its involvement in the central effects of vasopressin 1. Endocrinology 142: 1659-1668.
Hoekman JD, Ho RJ (2011). Enhanced analgesic responses after preferential delivery of morphine and fentanyl to the olfactory epithelium in rats. Anesth Analg 113: 641.

Hofmann SG, Fang A, Brager DN (2015). Effect of intranasal oxytocin administration on psychiatric symptoms: a meta-analysis of placebo-controlled studies. Psychiatry Res 228: 708-714.

Huang H, Michetti C, Busnelli M, Manago F, Sannino S, Scheggia D et al (2014). Chronic and acute intranasal oxytocin produce divergent social effects in mice. Neuropsychopharmacology 39: 1102-1114.

Huber D, Veinante P, Stoop R (2005). Vasopressin and oxytocin excite distinct neuronal populations in the central amygdala. Science 308: 245-248.

Insel TR (2010). The challenge of translation in social neuroscience: a review of oxytocin, vasopressin, and affiliative behavior. Neuron 65: 768-779.

Insel TR, O'Brien DJ, Leckman JF (1999). Oxytocin, vasopressin, and autism: is there a connection? Biol Psychiatry 45: 145-157.

Jocham Radua MD, Shergill SS (2015). Neurophysiological effects of acute oxytocin administration: systematic review and meta-analysis of placebo-controlled imaging studies. J Psychiatry Neurosci 40: E1.

Kalisch R, Elbel GK, Gossl C, Czisch M, Auer DP (2001). Blood pressure changes induced by arterial blood withdrawal influence bold signal in anesthesized rats at 7 Tesla: implications for pharmacologic MRI. Neuroimage 14: 891-898.

Kirkpatrick MG, Lee R, Wardle MC, Jacob S, de Wit H (2014). Effects of MDMA and intranasal oxytocin on social and emotional processing. Neuropsychopharmacology 39: 1654-1663.

Kirsch P, Esslinger C, Chen Q, Mier D, Lis S, Siddhanti S et al (2005). Oxytocin modulates neural circuitry for social cognition and fear in humans. J Neurosci 25: 11489-11493.

Kosaki Y, Watanabe S (2016). Conditioned social preference, but not place preference, produced by intranasal oxytocin in female mice. Behav Neurosci 130: 182-195.

Labuschagne I, Phan KL, Wood A, Angstadt M, Chua P, Heinrichs $\mathrm{M}$ et al (2010). Oxytocin attenuates amygdala reactivity to fear in generalized social anxiety disorder. Neuropsychopharmacology 35: 2403-2413.

Landgraf R, Ke+fler MS, Bunck M, Murgatroyd C, Spengler D, Zimbelmann $\mathrm{M}$ et al (2007). Candidate genes of anxiety-related behavior in $\mathrm{HAB} / \mathrm{LAB}$ rats and mice: focus on vasopressin and glyoxalase-I. Neurosci Biobehav Rev 31: 89-102.

Lee RJ, Coccaro EF, Cremers H, McCarron R, Lu SF, Brownstein MI et al (2013). A novel V1a receptor antagonist blocks vasopressininduced changes in the CNS response to emotional stimuli: an fMRI study. Front Syst Neurosci 7: 100.

Leng G, Ludwig M (2016). Intranasal oxytocin: myths and delusions. Biol Psychiatry 79: 243-250.

Litvin Y, Murakami G, Pfaff DW (2011). Effects of chronic social defeat on behavioral and neural correlates of sociality: vasopressin, oxytocin and the vasopressinergic V1b receptor. Physiol Behav 103: 393-403.

Logothetis NK (2008). What we can do and what we cannot do with fMRI. Nature 453: 869-878.

Ludwig M, Bull PM, Tobin VA, Sabatier N, Landgraf R, Dayanithi G et al (2005). Regulation of activity-dependent dendritic vasopressin release from rat supraoptic neurones. $J$ Physiol 564: 515-522.

Ludwig M, Tobin VA, Callahan MF, Papadaki E, Becker A, Engelmann $M$ et al (2013). Intranasal application of vasopressin fails to elicit changes in brain immediate early gene expression, neural activity and behavioural performance of rats. J Neuroendocrinol 25: 655-667.

Lukas M, Neumann ID (2013). Oxytocin and vasopressin in rodent behaviors related to social dysfunctions in autism spectrum disorders. Behav Brain Res 251: 85-94.

Lukas M, Toth I, Reber SO, Slattery DA, Veenema AH, Neumann ID (2011). The neuropeptide oxytocin facilitates pro- 
social behavior and prevents social avoidance in rats and mice. Neuropsychopharmacology 36: 2159-2168.

Luo F, Wu G, Li Z, Li S-J (2003). Characterisation of effects of mean arterial blood pressure induced by cocaine and cocaine methiodide on BOLD signals in the rat brain. Magn Reson Med 49: 264-270.

Mahmud M, Pasqualotto E, Bertoldo A, Girardi S, Maschietto M, Vassanelli S (2011). An automated method for detection of layer activation order in information processing pathway of rat barrel cortex under mechanical whisker stimulation. J Neurosci Methods 196: $141-150$.

Maier A, Wilke M, Aura C, Zhu C, Ye FQ, Leopold DA (2008). Divergence of fMRI and neural signals in V1 during perceptual suppression in the awake monkey. Nat Neurosci 11: 1193-1200.

Mandeville JB, Marota JJA, Kosofsky BE, Keltner JR, Weissleder R, Rosen B et al (1998). Dynamic functional imaging of relative cerebral blood volume during rat forepaw stimulation. Magn Reson Med 39: 615-624.

Marlin BJ, Mitre M, D'amour JA, Chao MV, Froemke RC (2015). Oxytocin enables maternal behaviour by balancing cortical inhibition. Nature 520: 499-504.

McCarthy MT, McDonald BC, Brooks PJ, Goldman D (1996). An anxiolytic action of oxytocin is enhanced by estrogen in the mouse. Physiol Behav 60: 1209-1215.

Mens WBJ, Witter A, Van Wimersma Greidanus TB (1983). Penetration of neurohypophyseal hormones from plasma into cerebrospinal fluid (CSF): half-times of disappearance of these neuropeptides from CSF. Brain Res 262: 143-149.

Meyer-Lindenberg A, Domes G, Kirsch P, Heinrichs M (2011). Oxytocin and vasopressin in the human brain: social neuropeptides for translational medicine. Nat Rev Neurosci 12: 524-538.

Modi ME, Connor-Stroud F, Landgraf R, Young LJ, Parr LA (2014). Aerosolized oxytocin increases cerebrospinal fluid oxytocin in rhesus macaques. Psychoneuroendocrinology 45: 49-57.

Neumann ID, Landgraf R (2012). Balance of brain oxytocin and vasopressin: implications for anxiety, depression, and social behaviors. Trends Neurosci 35: 649-659.

Neumann ID, Slattery DA (2016). Oxytocin in general anxiety and social fear: a translational approach. Biol Psychiatry 79: 213-221.

Neumann ID, Maloumby R, Beiderbeck DI, Lukas M, Landgraf R (2013). Increased brain and plasma oxytocin after nasal and peripheral administration in rats and mice. Psychoneuroendocrinology 38: 1985-1993.

Orth M, Bravo E, Barter L, Carstens E, Antognini JF (2006). The differential effects of halothane and isoflurane on electroencephalographic responses to electrical microstimulation of the reticular formation. Anesth Analg 102: 1709-1714.

Ostrowski NL, Lolait SJ, Young WS 3rd (1994). Cellular localization of vasopressin $\mathrm{V} 1 \mathrm{a}$ receptor messenger ribonucleic acid in adult male rat brain, pineal, and brain vasculature. Endocrinology 135: 1511-1528.

Owen SF, Tuncdemir SN, Bader PL, Tirko NN, Fishell G, Tsien RW (2013). Oxytocin enhances hippocampal spike transmission by modulating fast-spiking interneurons. Nature 500: 458-462.

Paloyelis Y, Doyle OM, Zelaya FO, Maltezos S, Williams SC, Fotopoulou A et al (2014). A spatiotemporal profile of in vivo cerebral blood flow changes following intranasal oxytocin in humans. Biol Psychiatry 79: 693-705.

Penagarikano O, Lzaro MT, Lu XH, Gordon A, Dong H, Lam HA et al (2015). Exogenous and evoked oxytocin restores social behavior in the Cntnap2 mouse model of autism. Sci Transl Med 7: 271ra278-271ra278.

Raggenbass M (2001). Vasopressin- and oxytocin-induced activity in the central nervous system: electrophysiological studies using in-vitro systems. Prog Neurobiol 64: 307-326.

Ramos L, Hicks C, Caminer A, McGregor IS (2014). Inhaled vasopressin increases sociability and reduces body temperature and heart rate in rats. Psychoneuroendocrinology 46: 46-51.
Riem MME, Bakermans-Kranenburg MJ, Voorthuis A, van IJzendoorn MH (2014). Oxytocin effects on mind-reading are moderated by experiences of maternal love withdrawal: an fMRI study. Prog Neuropsychopharmacol Biol Psychiatry 51: 105-112.

Ross HE, Young LJ (2009). Oxytocin and the neural mechanisms regulating social cognition and affiliative behavior. Front Neuroendocrinol 30: 534-547.

Ryckmans T (2010). Modulation of the vasopressin system for the treatment of CNS diseases. Curr Opin Drug Discov Dev 13: 538-547.

Sala M, Braida D, Donzelli A, Martucci R, Busnelli M, Bulgheroni E et al (2013). Mice heterozygous for the oxytocin receptor gene show impaired social behaviour but not increased aggression or cognitive inflexibility: evidence of a selective haploinsufficiency gene effect. J Neuroendocrinol 25: 107-118.

Sala M, Braida D, Lentini D, Busnelli M, Bulgheroni E, Capurro V et al (2011). Pharmacologic rescue of impaired cognitive flexibility, social deficits, increased aggression, and seizure susceptibility in oxytocin receptor null mice: a neurobehavioral model of autism. Biol Psychiatry 69: 875-882.

Saladin LK, Bruni JE (1993). The effects of intracerebroventricular versus intravenous administration of vasopressin on intracranial pressure in the rat. Neurol Res 15: 198-203.

Schridde U, Khubchandani M, Motelow JE, Sanganahalli BG, Hyder F, Blumenfeld H (2008). Negative BOLD with large increases in neuronal activity. Cereb Cortex 18: 1814-1827.

Sforazzini F, Schwarz AJ, Galbusera A, Bifone A, Gozzi A (2014). Distributed BOLD and CBV-weighted resting-state networks in the mouse brain. Neuroimage 87: 403-415.

Shalev I, Israel S, Uzefovsky F, Gritsenko I, Kaitz M, Ebstein RP (2011). Vasopressin needs an audience: neuropeptide elicited stress responses are contingent upon perceived social evaluative threats. Horm Behav 60: 121-127.

Shamay-Tsoory SG, Abu-Akel A (2016). The social salience hypothesis of oxytocin. Biol Psychiatry 79: 194-202.

Sharma V, McNeill JH (2009). To scale or not to scale: the principles of dose extrapolation. Br J Pharmacol 157: 907-921.

Squillace M, Dodero L, Federici M, Migliarini S, Errico F, Napolitano F et al (2014). Dysfunctional dopaminergic neurotransmission in asocial BTBR mice. Transl Psychiatry 4: e427.

Sripada CS, Phan KL, Labuschagne I, Welsh R, Nathan PJ, Wood AG (2013). Oxytocin enhances resting-state connectivity between amygdala and medial frontal cortex. Int J Neuropsychopharmacol 16: 255-260.

Stoop R (2012). Neuromodulation by oxytocin and vasopressin. Neuron 76: 142-159.

Stoop R (2014). Neuromodulation by oxytocin and vasopressin in the central nervous system as a basis for their rapid behavioral effects. Curr Opin Neurobiol 29: 187-193.

Takayanagi Y, Yoshida M, Bielsky IF, Ross HE, Kawamata M, Onaka $\mathrm{T}$ et al (2005). Pervasive social deficits, but normal parturition, in oxytocin receptor-deficient mice. Proc Natl Acad Sci USA 102: 16096-16101.

Thomas JS, Koh SH, Cooper GM (2007). Haemodynamic effects of oxytocin given as i.v. bolus or infusion on women undergoing Caesarean section. Br J Anaesth 98: 116-119.

US Food and Drug Administration (2005). Guidance for Industry: Estimating the Maximum Safe Starting Dose in Initial Clinical Trials for Therapeutics in Adult Healthy Volunteers. Center for Drug Evaluation and Research (CDER). Federal Register Vol 70, No. 140: 05-14456.

Veening JG, Olivier B (2013). Intranasal administration of oxytocin: behavioral and clinical effects, a review. Neurosci Biobehav Rev 37: 1445-1465.

Walum H, Waldman ID, Young LJ (2016). Statistical and methodological considerations for the interpretation of intranasal oxytocin studies. Biol Psychiatry 79: 251-257. 
Windle RJ, Shanks N, Lightman SL, Ingram CD (1997). Central oxytocin administration reduces stress-induced corticosterone release and anxiety behavior in rats 1. Endocrinology 138: 2829-2834.

Windle RJ, Kershaw YM, Shanks N, Wood SA, Lightman SL, Ingram CD (2004). Oxytocin attenuates stress-induced c-fos mRNA expression in specific forebrain regions associated with modulation of hypothalamo-pituitary-adrenal activity. J Neurosci 24: 2974-2982.

Yoshida M, Takayanagi Y, Inoue K, Kimura T, Young LJ, Onaka T et al (2009). Evidence that oxytocin exerts anxiolytic effects via oxytocin receptor expressed in serotonergic neurons in mice. J Neurosci 29: 2259-2271.
Young LJ, Barrett CE (2015). Can oxytocin treat autism? Science 347: 825-826.

Young WS, Li J, Wersinger SR, Palkovits M (2006). The vasopressin $1 \mathrm{~b}$ receptor is prominent in the hippocampal area CA2 where it is unaffected by restraint stress or adrenalectomy. Neuroscience 143: 1031-1039.

Zaharchuk G, Mandeville JB, Bogdanov AA Jr., Weissleder R, Rosen BR, Marota JJ (1999). Cerebrovascular dynamics of autoregulation and hypoperfusion. An MRI study of CBF and changes in total and microvascular cerebral blood volume during hemorrhagic hypotension. Stroke 30: 2197-2204.

Zink CF, Meyer-Lindenberg A (2012). Human neuroimaging of oxytocin and vasopressin in social cognition. Horm Behav 61: 400-409.

Supplementary Information accompanies the paper on the Neuropsychopharmacology website (http://www.nature.com/npp) 